Marinkovic, D. ; Rama, G.

\title{
Co-rotational shell element for numerical analysis of laminated piezoelectric composite structures
}

Journal article | Accepted manuscript (Postprint)

This version is available at https://doi.org/10.14279/depositonce-10665

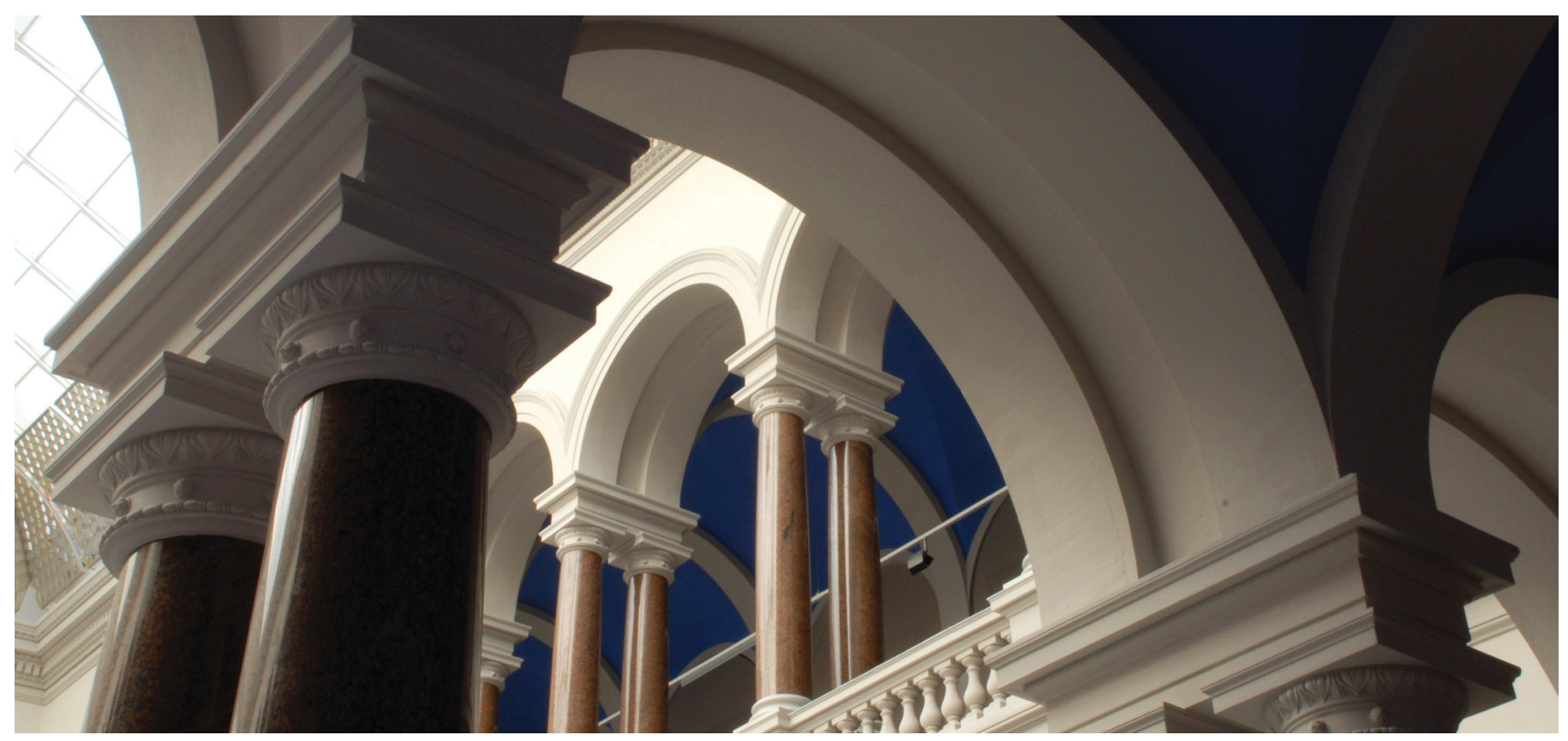

Marinković, D., \& Rama, G. (2017). Co-rotational shell element for numerical analysis of laminated piezoelectric composite structures. Composites Part B: Engineering, 125, 144-156.

https://doi.org/10.1016/j.compositesb.2017.05.061 


\title{
Co-rotational shell element for numerical analysis of laminated piezoelectric composite structures
}

\author{
Dragan Marinković ${ }^{1,2}$, Gil Rama ${ }^{1}$ \\ ${ }^{1}$ Department of Structural Analysis, Berlin Institute of Technology, Germany \\ Strasse des 17. Juni 135, 10623 Berlin (email: dragan.marinkovic@tu-berlin.de) \\ ${ }^{2}$ Faculty of Mechanical Engineering, University of Nis, Serbia
}

\begin{abstract}
Laminated composite structures consisting of load-carrying and multifunctional materials represent a rather powerful material system. The passive, load-carrying layers can be made of isotropic material or fiber-reinforced composites, while piezoelectric materials represent the most common choice of multifunctional materials for active layers. The multifunctionality of piezoelectric layers is provided by their inherent property to couple mechanical and electric fields. The property can thus be used to sense deformations or produce actuating forces. A highly efficient 3-node shell element is developed for modeling piezoelectric laminated composite shells. The equivalent single-layer approach and Mindlin-Reissner kinematics are used in the element formulation together with the discrete shear gap (DSG) technique to resolve the shear locking and strain smoothing technique to improve the performance. Piezoelectric layers are assumed to be polarized in the thickness direction thus coupling the in-plane strains with the electric field oriented in the thickness direction. The co-rotational FE formulation is used to account for geometrically nonlinear effects. Numerical examples cover linear and geometrically nonlinear static and dynamic cases with piezoelectric layers used as actuators and sensors.
\end{abstract}

Keywords: co-rotational FEM, piezoelectricity, fiber-reinforced laminates, actuator, sensor 


\section{INTRODUCTION}

Thin-walled structures render some $80 \%$ of all engineering structures and are still a growing portion of engineering structures in a broad range of sizes and quite diverse applications. They are a result of the tendency to reduce the structural dead-load but keep at the same time the high level of carrying capacity and stiffness. It is the combination of the shape and thinness of the walls that provides those advantages. The advantages are further improved by use of modern engineering materials - laminates, with layers made of various materials that could be isotropic or, more frequently, fiberreinforced composites. Orthotropic fiber-reinforced composite laminates offer vast options for tailoring material properties through the choice of constituent materials, fiber orientation, number, thickness and sequence of layers.

Despite all these advantages, composite laminates may also suffer from structural stability issues and are sensitive to vibrations. The idea behind the term smart/adaptive structures offers a great potential to cope with such challenges. The term has been adapted by the engineering community two decades ago to redefine the concept of structures from a conventional passive deformable system to an active controllable system with inherent self-sensing, diagnosis, actuation and control capabilities [1]. The use of multifunctional materials enables application of active elements (sensors and actuators) with excellent capability of structural integration. Piezoelectric materials represent quite a common choice of multifunctional materials for the considered type of structures, which is due to their operational frequency range as well as stroke and force range they can produce, when shaped for the use with thin-walled structures. Their inherent property to couple mechanical and electric fields is used for this purpose. Since it is a two-way coupling, it can be used for actuation by producing desired forces through a predefined electric potential (reverse piezoelectric effect), and for sensing, as 
deformations give rise to a strain-proportional electric field (direct piezoelectric effect). Such systems have a broad range of applicability, including vibration suppression [2, 3, 4], structural health monitoring [5, 6], shape control [7, 8], to name but a few.

Successful design of piezoelectric laminates and appropriate control laws calls for efficient and reliable approaches for modeling and simulation of their behavior. Whereas some researchers provided analytical considerations of piezoelectric laminated structures $[9,10,11]$, the attention was mainly turned to the finite element method (FEM) as a predominant numerical method in the field of structural analysis. Numerous developments of piezoelectric beam, plate and shell elements are the best prove of how enticing this research field is. An exhaustive overview would be prohibitively long. An interested reader may address the survey from Benjeddou [12] for a thorough overview of the development in the field during the ' 90 s and the development continued at the same pace in the years to come. Although it was confined to the considered type of material system and structures, still a few major streams of development can be distinguished. Some of the developments were aimed at high fidelity solid elements, with various techniques used to improve the performance. This includes the mixed variational formulation applied with an 8-node piezoelectric solid shell element by Klinkel and Wagner [13], and the assumed strain technique applied with an 18-node element by Lee et al. [14]. Willberg and Gabbert [15] applied the isogeometric approach to develop a 3D piezoelectric finite element for smart structures. Li at al. [16] proposed 2D and 3D elements with the smoothed strain technique for piezoelectric structures.

If the global structural behavior is aimed at, 2D elements, i.e. plates and shells, offer greater numerical efficiency. A large number of developed elements use the equivalent single-layer approach and are mainly based either on the classical laminate theory that implements Kirchoff-Love kinematics (e.g. [17, 18]), or the first-order shear deformation (FSDT) theory with Mindlin-Reissner kinematics. The latter was more 
frequently used in the FEM developments as it includes the transverse shear effects and requires the $\mathrm{C}^{0}$-continutity from the shape functions (compared to the $\mathrm{C}^{1}$-continuity needed for the classical laminate theory). The developments cover broad range of finite elements including linear triangular [19] and quadrilateral [20] shell elements, biquadratic 8-node [21] and 9-node [22] shell elements, etc. As shell elements are notorious for the shear and membrane locking phenomena, various techniques, such as discrete shear gap (DSG) [20], mixed-interpolation of tensorial components (MITC) [23], selectively [21] and uniformly [22] reduced integration, etc. were applied to alleviate the problem. The developed elements were used to investigate further effects in modeling electro-mechanical coupled field, such as the convergence behavior of FEM results [24], and, for users' convenience, some developments were also implemented in commercial FEM programs [25]. The isogeometric approach was also considered in the development of 2D elements for piezoelectric laminates. Phung-Van et al. [26] used it in combination with a higher-order shear deformation theory.

Layerweise theories were also addressed to provide finite elements that stand between the 2D elements based on the equivalent single-layer approach and 3D elements, regarding the numerical effort and achieved accuracy. For this purpose, the Carrera Unified Formulation (CUF) for multilayered plates and shells [27] is frequently applied. Based on it, Cinefra et al. [28] developed a 9-node plate element for static analysis using the MITC technique and variable through-the-thickness layer-wise kinematics. This development was later extended to cover free-vibration analyses of piezoelectric plates [29]. Milazzo [30] used the approach that reduces the coupled-field problem to mechanical one and implemented both equivalent single-layer and layerwise approaches.

Geometric nonlinearities were significantly less addressed in the available literature and this is one of the contributions this paper aims at. A linear triangular shell element, 
whose mechanical part is based on the development by Bletzinger et al. [31] and Nguyen-Thoi et al. [32], was extended by the authors of this article to include piezoelectric layers polarized in the thickness direction and to cover geometric nonlinearities characterized by finite local rotations but small strains. The co-rotational (CR) FEM formulation [33, 34, 35] is used for the purpose. Application of the element for static and dynamic actuator and sensor cases will be demonstrated.

\section{3-NODE PIEZOELECTRIC SHELL ELEMENT}

The choice to develop the linear triangular shell element was motivated by its high numerical efficiency and meshing ability. However, those advantages are accompanied by the disadvantage of relatively stiff element behavior. Since it is a flat element, shell behavior is obtained by directly superposing the plate and membrane behavior. The mechanical field of the element relies on the development by Bletzinger et al. [31] and the DSG technique is used to alleviate the shear locking. Nguyen-Thoi et al. [32] used the strain smoothing technique to further improve this element, i.e. to avoid large strain and stress oscillations between adjacent elements and to render the element formulation independent from node numbering. Another aspect that talks in favor of the linear triangular element is the objective of its implementation into the co-rotational FEM formulation for geometrically nonlinear analysis. Since the rigid-body rotation is considered element-wise (one rotation matrix per element), finer meshes are needed for adequate accuracy regardless of the element properties. In what follows, only the basic equations that describe the element mechanical and electric fields are given.

\subsection{Element geometry and mechanical field}

Two coordinate systems are used in the element formulation - the global $(\mathrm{x}, \mathrm{y}, \mathrm{z})$ and local $\left(x^{\prime}, y^{\prime}, z^{\prime}\right)$ coordinate systems. The local coordinate system is essential for the description of element geometry, implementation of kinematics and constitutive equations 
but also for the description of the electric field and piezoelectric coupling. It is defined so as to have one of its axes, the $\mathrm{x}^{\prime}$-axis, oriented from element node 1 towards node 2 , while the $z^{\prime}$-axis is perpendicular to the element surface, Fig. 1. It takes very basic vector algebra to define the unit vectors of the local coordinate system, $\left\{e_{x^{\prime}}\right\},\left\{e_{y^{\prime}}\right\}$ and $\left\{e_{z^{\prime}}\right\}$ and this is omitted here for the sake of brevity.

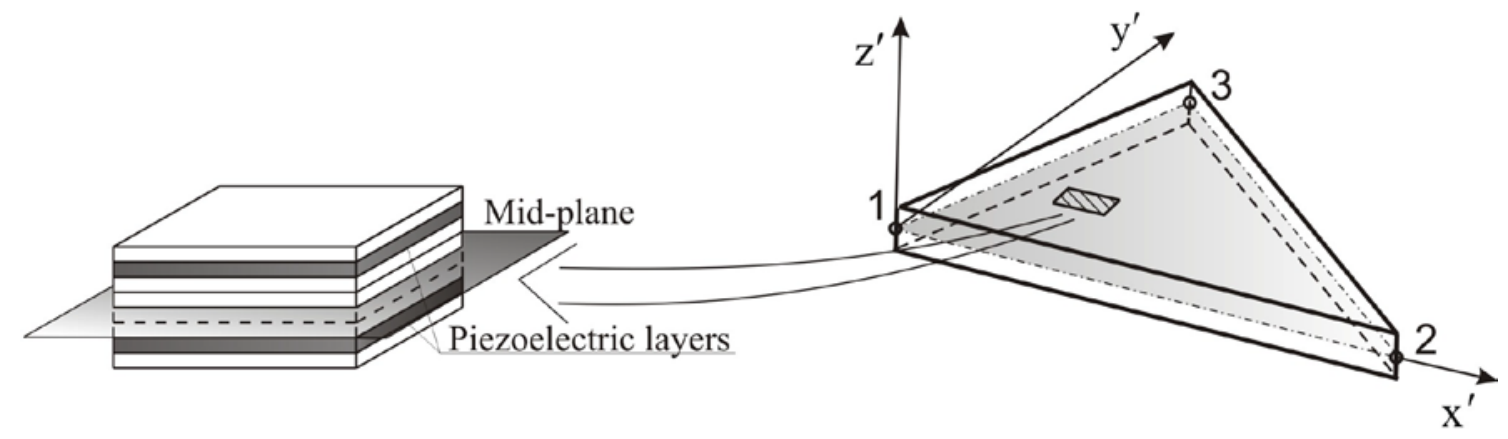

Fig. 1 Geometry, local coordinate system and material system of the 3-node shell element

The element uses linear shape functions which are defined in a manner common for triangular elements. Any point within the element, with local coordinates $\mathrm{x}^{\prime}$ and $\mathrm{y}^{\prime}$, forms 3 sub-triangles in the element. The shape function of node $i$ at any point $\left(x^{\prime}, y^{\prime}\right)$ in the element domain is defined as a ratio of the corresponding sub-triangle surface area (defined by the point and the remaining two element nodes) and the element surface area. Hence, the shape functions for all 3 element nodes read:

$$
\begin{aligned}
& N_{1}\left(x^{\prime}, y^{\prime}\right)=\frac{1}{2 A_{e}}\left[\left(x_{1}^{\prime} y_{3}^{\prime}-x_{3}^{\prime} y_{2}^{\prime}\right)+\left(y_{2}^{\prime}-y_{3}^{\prime}\right) x^{\prime}+\left(x_{3}^{\prime}-x_{2}^{\prime}\right) y^{\prime}\right] \\
& N_{2}\left(x^{\prime}, y^{\prime}\right)=\frac{1}{2 A_{e}}\left[\left(x_{3}^{\prime} y_{1}^{\prime}-x_{1}^{\prime} y_{3}^{\prime}\right)+\left(y_{3}^{\prime}-y_{1}^{\prime}\right) x^{\prime}+\left(x_{1}^{\prime}-x_{3}^{\prime}\right) y^{\prime}\right] \\
& N_{3}\left(x^{\prime}, y^{\prime}\right)=\frac{1}{2 A_{e}}\left[\left(x_{1}^{\prime} y_{2}^{\prime}-x_{2}^{\prime} y_{1}^{\prime}\right)+\left(y_{1}^{\prime}-y_{2}^{\prime}\right) x^{\prime}+\left(x_{2}^{\prime}-x_{1}^{\prime}\right) y^{\prime}\right]
\end{aligned}
$$


with $x_{i}^{\prime}$ and $y^{\prime}{ }_{i}, i=1,2,3$ denoting the local coordinates of the element nodes, while $A_{e}$ is the element surface area. The shell thickness is assumed to be perpendicular to the midsurface and the element geometry with respect to the local coordinate system is given as:

$$
\left\{\begin{array}{l}
x^{\prime} \\
y^{\prime} \\
z^{\prime}
\end{array}\right\}=\sum_{i=1}^{3} N_{i}\left\{\begin{array}{c}
x_{i}^{\prime} \\
y_{i}^{\prime} \\
0
\end{array}\right\}+\sum_{i=1}^{3} \frac{h}{2} N_{i} \xi\left\{e_{z^{\prime}}\right\}
$$

where $h$ denotes the shell thickness and the natural coordinate in the thickness direction takes values $-1<\xi<+1$.

The Mindlin-Reissner kinematics implies that the displacement of any point $\mathrm{P}$ of the element is represented as a superposition of the displacement of the corresponding midsurface point, $\mathrm{P}_{0}$, and the relative displacement of $\mathrm{P}$ with respect to $\mathrm{P}_{0}$. The latter is the consequence of thickness line rotations $\theta_{x^{\prime}}$ and $\theta_{y^{\prime}}$ at the mid-surface point $\mathrm{P}_{0}$ around the $x^{\prime}$ - and $y^{\prime}$-axes, respectively. Hence, the displacement field $\left\{u^{\prime}, v^{\prime}, w^{\prime}\right\}^{\mathrm{T}}$ with respect to the local coordinate system is obtained as:

$$
\left\{\begin{array}{c}
u^{\prime} \\
v^{\prime} \\
w^{\prime}
\end{array}\right\}=\sum_{i=1}^{3} N_{i}\left\{\begin{array}{c}
u_{i}^{\prime} \\
v_{i}^{\prime} \\
w_{i}^{\prime}
\end{array}\right\}+\sum_{i=1}^{3} \frac{h}{2} N_{i} \xi\left\{\begin{array}{c}
\theta_{y^{\prime} i} \\
-\theta_{x^{\prime} i} \\
0
\end{array}\right\}
$$

where the subscripting $i$ denotes a nodal value at node $i$.

In a manner typical for shells, the strain field is divided into the membrane-flexural, i.e. in-plane strains, $\left\{\varepsilon_{m f}^{\prime}\right\}$, and the transverse shear strains, $\left\{\varepsilon_{s}^{\prime}\right\}$ with respect to the local coordinate system. They are defined by the corresponding strain-displacement matrices, $\left[B_{m f}\right]$ and $\left[B_{s}\right]$, respectively:

$$
\left\{\varepsilon^{\prime}\right\}=\left\{\begin{array}{c}
\left\{\varepsilon_{m f}^{\prime}\right\} \\
-- \\
\left\{\varepsilon_{s}^{\prime}\right\}
\end{array}\right\}=\left[\begin{array}{c}
{\left[B_{m f}\right]} \\
-- \\
{\left[B_{s}\right]}
\end{array}\right]\left\{d^{\prime}\right\}=\left[B_{u}\right]\left\{d^{\prime}\right\}
$$


where $\left\{d_{i}{ }^{\prime}\right\}$ is the vector of nodal translations and rotations.

The idea behind the discrete shear gap approach is the separation of deformation into a part due to transverse shear and a part due to bending. The shear 'gap' represents the difference between the total deformation and deformation due to bending. This distinction allows to handle separately the shear 'gap' and since it is done at discrete points, the term 'discrete shear gap' was introduced. An interested reader is referred to [31] for more details on the derivation of the strain-displacement matrices $\left[B_{m f}\right]$ and $\left[B_{s}\right]$ within the framework of the DSG approach. Thus, without going into the details, the matrices are given here directly:

$$
\begin{gathered}
{\left[B_{m f, i}\right]=\left[\begin{array}{ccccc}
N_{i, x^{\prime}} & 0 & 0 & N_{i, x^{\prime}} & 0 \\
0 & N_{i, y^{\prime}} & 0 & -N_{i, y^{\prime}} & 0 \\
N_{i, y^{\prime}} & N_{i, x^{\prime}} & 0 & -N_{i, x^{\prime}} & N_{i, y^{\prime}}
\end{array}\right]} \\
{\left[B_{s, 1}\right]=\frac{1}{2 A_{e}}\left[\begin{array}{ccccc}
0 & 0 & b-c & 0 & -A_{e} \\
0 & 0 & d-a & A_{e} & 0
\end{array}\right]} \\
{\left[B_{s, 2}\right]=\frac{1}{2 A_{e}}\left[\begin{array}{ccccc}
0 & 0 & c & -b c / 2 & a c / 2 \\
0 & 0 & -d & b d / 2 & -a d / 2
\end{array}\right]} \\
{\left[B_{s, 3}\right]=\frac{1}{2 A_{e}}\left[\begin{array}{ccccc}
0 & 0 & -b & b c / 2 & b d / 2 \\
0 & 0 & a & -a c / 2 & a d / 2
\end{array}\right]}
\end{gathered}
$$

where $N_{i, x^{\prime}}$ and $N_{i, y^{\prime}}, i=1,2,3$, are the constant derivatives of the shape functions in the local element coordinate system, while $a, b, c$ and $d$ read:

$$
\begin{array}{ll}
a=x_{2}^{\prime}-x_{1}^{\prime} & b=y_{2}^{\prime}-y_{1}^{\prime} \\
c=y_{3}^{\prime}-y_{1}^{\prime} & d=x_{3}^{\prime}-x_{1}^{\prime}
\end{array}
$$

Furthermore, the element is improved by the strain smoothing technique [32]. The basic idea of the technique is very simple. The element domain is divided into 3 subtriangles using the element centroid O (Fig. 2). 


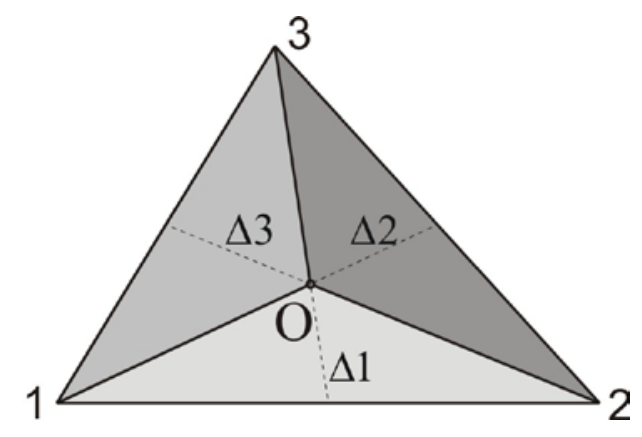

Fig. 2 Division of the triangular shell element into three sub-triangles

The strain-displacement matrices are computed for each of the sub-triangles according to Eqs. (5) and (6). Additionally, the displacement at the element centroid O, which is the common node of the 3 sub-triangles is assumed as the average value of the displacements at the 3 element nodes. Introducing this relation for the centroid displacement into the strain-displacement matrices and upon rearrangement of the terms, the resulting strain-displacement matrices for the sub-triangles are:

$$
\begin{aligned}
& {\left[\begin{array}{c}
{\left[B_{m f}^{\Delta 1}\right]} \\
-- \\
{\left[B_{s}^{\Delta 1}\right]}
\end{array}\right]=\left[\begin{array}{ccc}
{\left[\frac{1}{3}\left[B_{m f, 1}^{\Delta 1}\right]+\left[B_{m f, 2}^{\Delta 1}\right]\right]} & {\left[\frac{1}{3}\left[B_{m f, 1}^{\Delta 1}\right]+\left[B_{m f, 3}^{\Delta 1}\right]\right.} & \frac{1}{3}\left[B_{m f, 1}^{\Delta 1}\right] \\
{\left[\frac{1}{3}\left[B_{s, 1}^{\Delta 1}\right]+\left[B_{s, 2}^{\Delta 1}\right]\right]} & {\left[\frac{1}{3}\left[B_{s, 1}^{\Delta 1}\right]+\left[B_{s, 3}^{\Delta 1}\right]\right]} & \frac{1}{3}\left[B_{s, 1}^{\Delta 1}\right]
\end{array}\right]}
\end{aligned}
$$

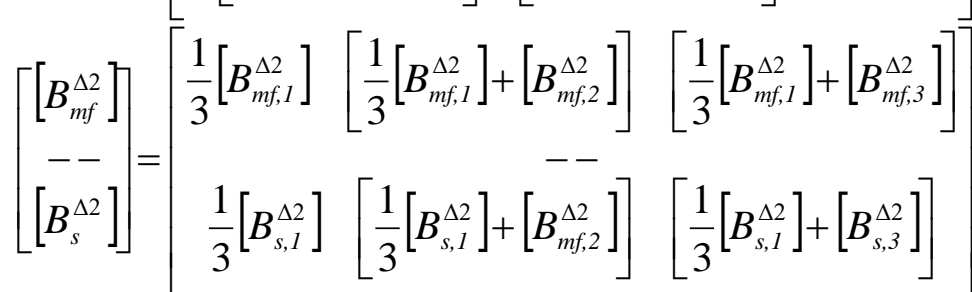

$$
\begin{aligned}
& {\left[\begin{array}{c}
{\left[B_{m f}^{\Delta 3}\right]} \\
-- \\
{\left[B_{s}^{\Delta 3}\right]}
\end{array}\right]=\left[\begin{array}{ccc}
{\left[\frac{1}{3}\left[B_{m f, 1}^{\Delta 3}\right]+\left[B_{m f, 3}^{\Delta 3}\right]\right]} & \frac{1}{3}\left[B_{m f, 1}^{\Delta 3}\right] & {\left[\frac{1}{3}\left[B_{m f, 1}^{\Delta 3}\right]+\left[B_{m f, 2}^{\Delta 3}\right]\right.} \\
{\left[\frac{1}{3}\left[B_{s, 1}^{\Delta 3}\right]+\left[B_{s, 3}^{\Delta 3}\right]\right]} & \frac{1}{3}\left[B_{s, 1}^{\Delta 3}\right] & {\left[\frac{1}{3}\left[B_{s, 1}^{\Delta 3}\right]+\left[B_{s, 2}^{\Delta 3}\right]\right.}
\end{array}\right]}
\end{aligned}
$$


where $\left[B_{m f, i}^{\Delta j}\right],\left[B_{s, i}^{\Delta j}\right]$ are the nodal strain displacement matrices of the $j^{\text {th }}$ sub-triangle's $i^{\text {th }}$ node. Simple averaging of the matrices in Eqs. (8) yields the element smoothed straindisplacement matrix:

$$
\left[\begin{array}{c}
{\left[B_{m f^{*}}\right.} \\
-- \\
{\left[B_{s^{*}}\right]}
\end{array}\right]=\frac{1}{3} \sum_{i=1}^{3}\left[\begin{array}{c}
{\left[B_{m f}^{\Delta i}\right.} \\
-- \\
{\left[B_{s}^{\Delta i}\right]}
\end{array}\right]
$$

Computation of the element mechanical stiffness matrix typically requires integration. However, the element strain-displacement matrix is constant over the element domain and the only integration needed is in the thickness direction. The user's input includes the material properties of each layer with respect to the material orientation (fiber orientation) together with the angle between the material orientation of the layer and the structural reference orientation. Hence, in the first step, the plane stress assumption is implemented to obtain the reduced material properties, which are further (in-plane) rotated to the structural reference direction and, finally, integrated in the thickness direction to yield the well-known ABD matrix for laminates [36]. The element mechanical stiffness matrix in the local coordinate system is then computed directly as:

$$
\left[K_{e}^{\prime}\right]=A_{e}\left(\left[\begin{array}{c}
{\left[B_{m f^{*}}\right.} \\
-- \\
{\left[B_{s^{*}}\right]}
\end{array}\right]^{\mathrm{T}}\left[\begin{array}{cc}
{[A]} & {[B]} \\
{[B]} & {[D]}
\end{array}\right]\left[\begin{array}{c}
{\left[B_{m f^{*}}\right.} \\
-- \\
{\left[B_{s^{*}}\right]}
\end{array}\right]\right)
$$

and using the transformation matrix between the global and local coordinate systems, $[T]=\left[\left\{e_{x^{\prime}}\right\}\left\{e_{y^{\prime}}\right\}\left\{e_{z^{\prime}}\right\}\right]$, the element mechanical stiffness matrix in the global coordinate system is obtained in a straightforward manner:

$$
\left[K_{e}\right]=[T]^{\mathrm{T}}\left[K_{e}^{\prime}\right][T]
$$




\subsection{Electric field and piezoelectric coupling}

The form of the constitutive equations of the piezoelectric material depends on the choice of independent variables. In the FEM formulation those are the mechanical displacements and electric potential and hence, the adequate form of the constitutive equations reads [37]:

$$
\begin{aligned}
& \{\sigma\}=\left[C^{E}\right]\{\varepsilon\}-[e]\{E\} \\
& \{D\}=[e]\{\varepsilon\}+\left[d^{\varepsilon}\right]\{E\}
\end{aligned}
$$

where $\{\sigma\}$ is the mechanical stress (Voigt notation), $\{D\}$ is the electric displacement vector, $\left[C^{E}\right]$ the piezoelectric material Hooke's matrix at constant electric field $\{E\},\left[d^{E}\right]$ the dielectric permittivity matrix at constant strain $\{\varepsilon\}$, and $[e]$ denotes the piezoelectric coupling matrix.

When applied to thin-walled laminated structures, piezoelectric sensors and actuators are typically shaped as thin patches polarized in the thickness direction and operating based on the e31-effect, which couples the electric field in the thickness direction with the in-plane strains. Such patches can be either embedded in the laminate or bonded to the outer surfaces. The patches are covered with electrodes on both surfaces so that an electric voltage that is constant over the piezoelectric patch surface is either supplied to the patch (actuator) or induced due to deformation (sensor).

The electric potential, $\varphi$, and electric field, $E$, in the piezoelectric layer are related in the well-known way:

$$
E=-\frac{\partial \varphi}{\partial \mathbf{z}^{\prime}}
$$

The functions for the electric potential and electric field that would satisfy the Gauss law are strongly related to the theory used to describe the kinematics. The conducted 
researches [38, 39] demonstrated that a consistent fulfillment of the Gauss law for dielectrics (piezoelectric material) in bending would require a quadratic function for the electric potential and a linear function for the electric potential across the thickness. However, the same researches also showed that typical approximations that use a linear function for the electric potential and a constant electric field are sufficiently accurate for typical thin piezoelectric patches, Fig 3. For that reason, they are also adapted in this work, leading to:

$$
E_{k}=-\frac{\Delta \Phi_{k}}{h_{k}}
$$

where $\Delta \Phi_{k}$ is the electric potential difference between the electrodes of the piezoelectric layer (say, $k^{\text {th }}$ layer) and $h_{k}$ is the thickness of the piezolayer. The resulting electric field - electric potential matrix, $\left[B_{\phi}\right]$, is diagonal with $1 / h_{k}$ as a typical main diagonal element:

$$
\left[B_{\phi}\right]=\left[\begin{array}{lll}
\ddots & & \\
& \frac{1}{h_{k}} & \\
& & \ddots
\end{array}\right]
$$

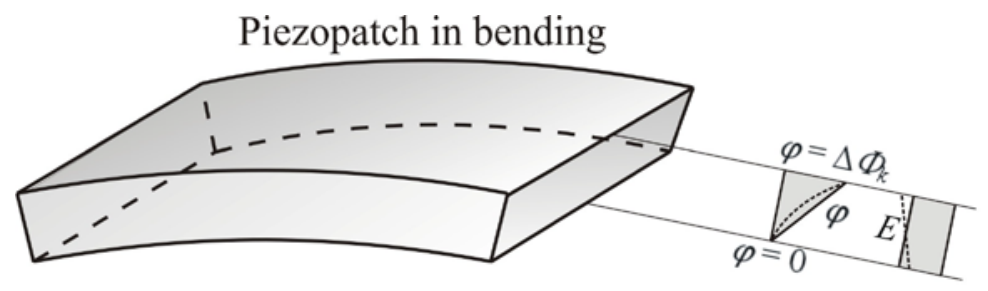

Fig. 3 Electric field and electric potential in the piezoelectric patch - accurate (dashed lines) and approximate (solid lines) functions in bending 
With the strain-displacement and electric field-electric potential matrices, the element inverse and direct piezoelectric coupling stiffness matrices, $\left[K_{u \phi}\right]$ and $\left[K_{\phi u}\right]$, are computed in the following manner:

$$
\left[K_{u \phi}\right]=\int_{V}\left(\left[B_{m f^{*}}\right]^{\mathrm{T}}\left[e^{\prime}\right]\left[B_{\phi}\right]\right) d V=\left[K_{\phi u}\right]^{\mathrm{T}}
$$

where only the membrane-flexural part of the strain-displacement matrix, $\left[B_{m f *}\right]$, is used because of the assumption that the applied piezopatches couple only the in-plane (membrane and flexural) strains with the electric field in the thickness direction, while $\left[e^{\prime}\right]$ is the matrix of the piezoelectric constants reduced in accordance with the assumption of zero normal stress in the thickness direction [38]. It should be noticed that the inverse and direct piezoelectric coupling matrices are transpose of each other. The number of rows of the inverse piezoelectric matrix $\left[K_{u \phi}\right]$ equals the number of mechanical degrees of freedom $(3 \times 5=15)$ and the number of columns equals the number of piezoelectric layers in the area of the structure covered by the finite element.

Finally, the dielectric stiffness matrix is computed as follows:

$$
\left[K_{\varphi \varphi}\right]=-\int_{V}\left[B_{\varphi}\right]^{T}\left[d^{\varepsilon}\right]\left[B_{\varphi}\right] d V
$$

and it is a square diagonal matrix with the number of rows and columns equal to the number of piezoelectric layers in the area of the structure covered by the finite element.

\section{FINITE ELEMENT EQUATIONS AND THE CO-ROTATIONAL APPROACH}

The dynamic FE equations for the piezoelectric continuum can be derived from the Hamilton's principle [40]. In geometrically nonlinear analysis, the structural 
configuration is continuously updated and for an implicit time integration scheme [41] the equations read:

$$
\begin{aligned}
& {\left[\begin{array}{cc}
{\left[M_{u u}\right]} & {[0]} \\
{[0]} & {[0]}
\end{array}\right]\left\{\begin{array}{c}
{ }^{t+\Delta t}\{\ddot{u}\}^{(k)} \\
\{0\}
\end{array}\right\}+\left[\begin{array}{rr}
{ }^{t+\Delta t}\left[C_{u u}\right] & {[0]} \\
{[0]} & {[0]}
\end{array}\right\}\left\{\begin{array}{r}
{ }^{t+\Delta t}\{\dot{u}\} \\
\{0\}
\end{array}\right\}+\left[\begin{array}{rl}
{ }^{t}\left[K_{u u}\right] & { }^{t}\left[K_{u \phi}\right] \\
{ }^{t}\left[K_{\phi u}\right] & { }^{t}\left[K_{\phi \phi}\right]
\end{array}\right]\left\{\begin{array}{l}
{ }^{t}\{\Delta u\}^{(k)} \\
{ }^{t}\{\Delta \phi\}^{(k)}
\end{array}\right\}} \\
& =\left\{\begin{array}{c}
{ }^{t+\Delta t}\left\{F_{\text {ext }}\right\}-{ }^{t+\Delta t}\left\{F_{\text {in }}\right\}^{(k-1)} \\
{ }^{t+\Delta t}\left\{Q_{\text {ext }}\right\}-{ }^{t+\Delta t}\left\{Q_{\text {in }}\right\}^{(k-1)}
\end{array}\right\}
\end{aligned}
$$

where $\left[M_{u u}\right]$ is the global mass matrix, $\left[C_{u u}\right]$ the mechanical damping matrix, $\left[K_{u u}\right]$, $\left[K_{u \phi}\right],\left[K_{\phi u}\right]$ and $\left[K_{\phi \phi}\right]$ are the global mechanical stiffness, piezoelectric direct and inverse coupling, and dielectric stiffness matrices, respectively, while the vectors $\{\Delta \phi\}$, $\{\Delta u\},\{\dot{u}\},\{\ddot{u}\}$ comprise the incremental differences of electric potentials of the piezolayers, incremental displacements, nodal velocities and accelerations, respectively. The vectors $\left\{F_{e x t}\right\},\left\{F_{i n}\right\},\left\{Q_{e x t}\right\}$ and $\left\{Q_{i n}\right\}$ on the right hand-side of the FE equations are the external and internal mechanical forces and electric charges, respectively. The time at which a quantity is taken is denoted by the left superscript, while the index $(k)$ in the right superscript denotes the iteration number, since the solution proceeds iteratively.

Geometric nonlinearities are accounted for in the present development using the corotational (CR) formulation. The basic idea is that each element has a local reference frame that is attached to the element and performs the same rigid-body motion as the element, Fig. 4. In this way, it is possible to decompose the overall element motion into a rigid-body motion and deformable motion. Upon extraction of the rigid-body motion from the overall motion, one obtains the nodal displacements and rotations that cause pure deformation. They are further used to compute internal forces and moments. Generally speaking, different material particles of a structure exhibit different rigidbody rotations throughout the deformation. The rigid-body rotation can be determined 
by polar decomposition of the deformation gradient. In case of the linear triangular element, the deformation gradient is constant over the whole element domain, thus yielding a constant rotation matrix per element. For more complex elements a kind of average rotation matrix is to be determined.

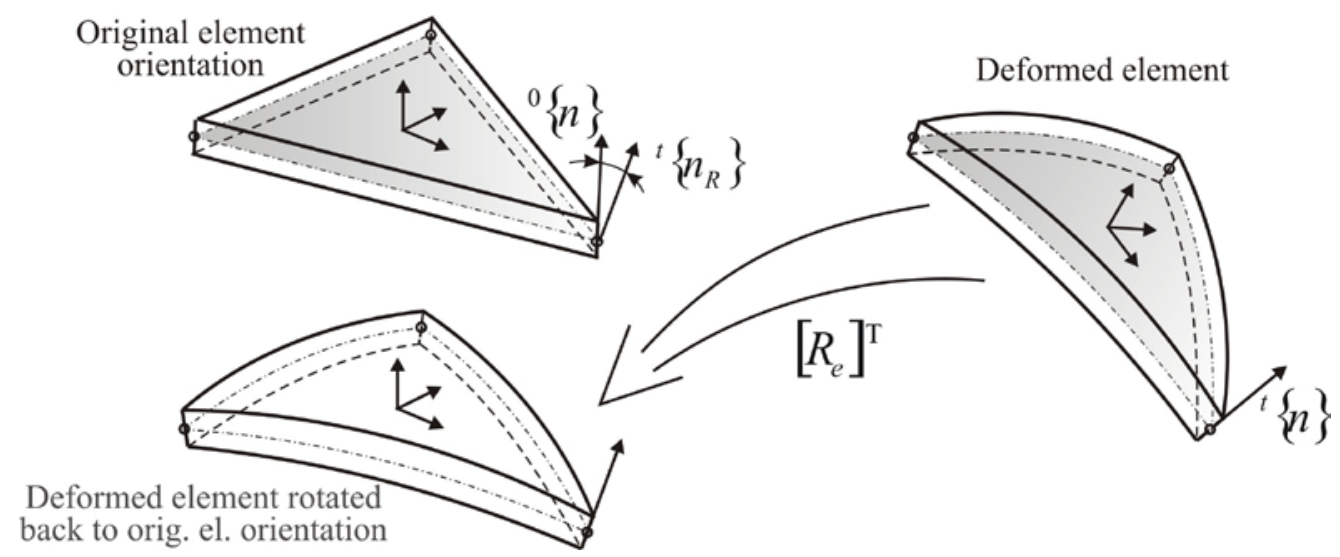

Fig. 4 Co-rotational approach

Once the element rotation matrix between the initial and current element configurations, ${ }^{t}[R]$, is determined, the element stiffness matrix is updated as follows:

$$
{ }^{t}\left[K_{e}\right]={ }^{t}\left[R_{e}\right]^{0}\left[K_{e}\right]^{t}\left[R_{e}\right]^{\mathrm{T}}
$$

The nodal rotation-free translations ${ }^{t}\left\{u_{T, i}^{r}\right\}$ with respect to the original element orientation are then calculated by:

$$
{ }^{t}\left\{u_{T, i}^{r}\right\}=\left\{\begin{array}{c}
{ }^{t} u_{i}^{r} \\
{ }^{t} v_{i}^{r} \\
{ }^{t} w_{i}^{r}
\end{array}\right\}={ }^{t}\left[R_{e}\right]^{\mathrm{T}}\left\{\begin{array}{c}
{ }^{t} x_{i} \\
{ }^{t} y_{i} \\
{ }^{t} z_{i}
\end{array}\right\}-\left\{\begin{array}{c}
x_{i} \\
y_{i} \\
z_{i}
\end{array}\right\}
$$

where $x_{i}, y_{i}$, and $z_{i}, i=1,2,3$, are the global coordinates of the element nodes.

Computation of deformational nodal rotations is somewhat more demanding. It is done by updating the nodal shell normals by means of incremental nodal rotations. To 
do this, the incremental rotation matrix of a shell normal at a node $i$ is determined by the following set of equations [42]:

$$
\begin{gathered}
{ }^{t-\Delta t}\left[Q_{i}\right]=[I]+\frac{\sin ^{t-\Delta t} \gamma_{i}}{{ }^{t-\Delta t} \gamma_{i}}{ }^{t-\Delta t}[S]+\frac{1}{2}\left(\frac{\sin \left({ }^{t-\Delta t} \gamma_{i} / 2\right)}{\left({ }^{t-\Delta t} \gamma_{i} / 2\right)}\right)^{2}{ }^{t-\Delta t}\left[S_{i}\right]^{2} \\
{ }^{t-\Delta t} \gamma_{i}=\sqrt{{ }^{t-\Delta t} \Delta \theta_{i 1}^{2}+{ }^{t-\Delta t} \Delta \theta_{i 2}^{2}+{ }^{t-\Delta t} \Delta \theta_{i 3}^{2}} \\
{ }^{t-\Delta t}\left[S_{i}\right]=\left[\begin{array}{ccc}
0 & -{ }^{t-\Delta t} \Delta \theta_{i 3} & { }^{t-\Delta t} \Delta \theta_{i 2} \\
{ }^{t-\Delta t} \Delta \theta_{i 3} & 0 & -{ }^{t-\Delta t} \Delta \theta_{i 1} \\
-{ }^{t-\Delta t} \Delta \theta_{i 2} & { }^{t-\Delta t} \Delta \theta_{i 1} & 0
\end{array}\right]
\end{gathered}
$$

where ${ }^{t-\Delta t} \Delta \theta_{i 1},{ }^{t-\Delta t} \Delta \theta_{i 2}$ and ${ }^{t-\Delta t} \Delta \theta_{i 3}$ are the three incremental global nodal rotations between the configurations at times $t-\Delta t$ and $t$, and the index $i$ pertains to node number. The nodal shell normal is updated using the rotation matrix ${ }^{t-\Delta t}\left[Q_{i}\right]$, hence:

$$
{ }^{t}\left\{n_{\mathrm{i}}\right\}={ }^{t-\Delta t}\left[Q_{i}\right]^{t-\Delta t}\left\{n_{\mathrm{i}}\right\}
$$

In the next step, the element rotation matrix ${ }^{t}\left[R_{e}\right]$, is used to rotate the current node normal backwards to the initial element configuration:

$$
{ }^{t}\left\{n_{R i}\right\}={ }^{t}\left[R_{e}\right]^{\mathrm{T}}{ }^{t}\left\{n_{i}\right\}
$$

and finally, the angle between the rotated normal , ${ }^{t}\left\{n_{R i}\right\}$, and the original normal, ${ }^{0}\left\{n_{i}\right\}$, yields the deformational nodal rotations, Fig. 4. Hence, the computed nodal translations and rotations, both free of rigid-body rotations, are used together with the linear stiffness matrix to compute the internal forces and moments. With the update stiffness matrix and internal forces and moments, the geometrically nonlinear FE computation may proceed. 


\section{NUMERICAL EXAMPLES}

In the following, examples are studied covering static and dynamic cases of relatively simple structures that involve composite laminates with different types of passive layers as well as active piezoelectric layers in both sensor and actuator modes. Linear and geometrically nonlinear computations are performed.

\subsection{Active beam with two pairs of piezo-actuators}

In the first example a clamped beam with two pairs of piezoelectric patches along its length is considered. Observing each pair of piezopatches separately, the polarization in patches is opposite so that a voltage applied across the thickness tends to induce (opposite) strains in the patches through the inverse piezoelectric effect. Since the patches are restrained (glued to the beam surface), equivalent stresses are induced resulting in uniformly distributed bending moments along the edges of the piezopatches. Obviously, two oppositely polarized piezopatches amplify the effect resulting from the piezoelectric coupling and this is the reason why this configuration is often used in such applications. The passive material of the beam is aluminum ( $Y=63.8 \mathrm{GPa}, \quad v=0.345)$, while the piezoelectric patches have the following material properties: $Y_{1}=47.6 \mathrm{GPa}$, $v=0.3, e_{31}=e_{32}=18.02 \mathrm{C} / \mathrm{m}^{2}$. The length of the beam is $300 \mathrm{~mm}$, width $25 \mathrm{~mm}$ and thickness $0.645 \mathrm{~mm}$. The piezopatches are of the same width as the beam itself, their length is $25 \mathrm{~mm}$ and the thickness of each piezopatch is $0.25 \mathrm{~mm}$, so that the overall thickness in the areas covered by piezopatches is $1.145 \mathrm{~mm}$. The beam geometry and position of the two pairs of piezopatches are depicted in Fig. 5.

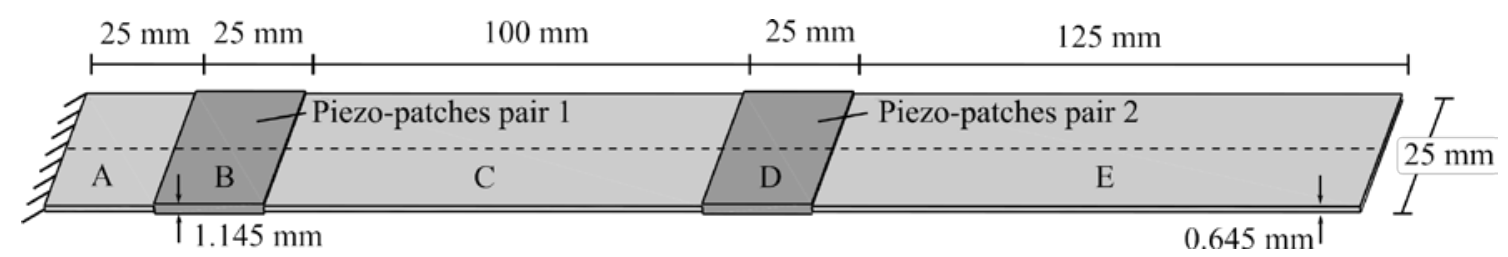

Fig. 5 Geometry of the active beam with two pairs of oppositely polarized patches 
The supplied voltage to the piezopatches of the first pair is $100 \mathrm{~V}$, while for the second pair it is $-100 \mathrm{~V}$. As already mentioned, this gives rise to bending moments uniformly distributed along the edges of the piezopatches, but the bending moments are oppositely oriented in the two pairs of piezopatches. Hence, the areas covered by piezopatches are exposed to pure bending and since the patches are of the same size and polarization, as well as exposed to the same electric voltages, only with opposite sign, they exhibit he same bending deformation but with opposite curvatures. Observing the width mid-line along the beam length (dashed line in Fig. 5), it is to be expected that it remains undeformed in area A, bends in areas B and D with opposite curvatures, exhibits pure rotation in area $\mathrm{C}$ and remains undeformed but translated in z-direction in area E. The diagram in Fig. 6, which depicts the beam deflection along the length, confirms this expectation. The results are obtained using the FE mesh with 288 triangular elements. Due to small deformations linear analysis was performed. Figure 6 also comprises experimental results for this case reported by Gupta et al. [43]. Obviously, the reported measurement results for the beam deflection feature some scattering, which is to be attributed to the measurement errors. Despite of that, the numerical result by the present element is in good agreement with the experimental result.

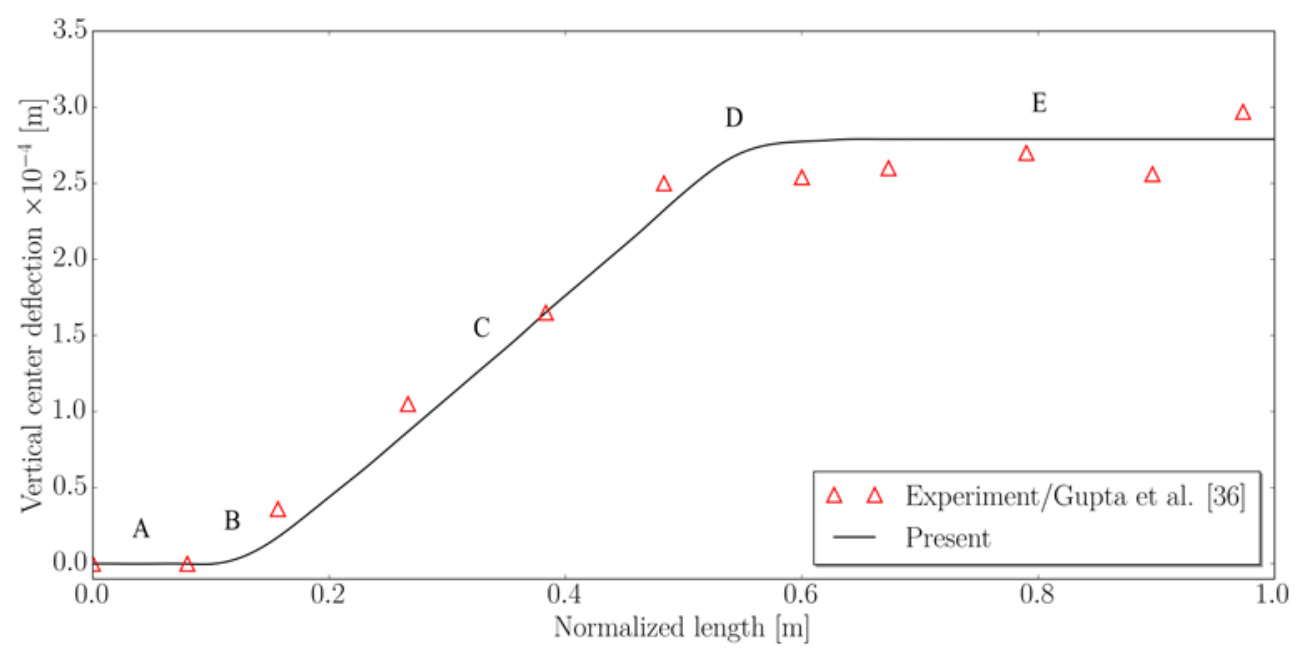

Fig. 6 Active beam deflection upon actuation 


\subsection{Active plate with multiple actuators}

The following example was originally proposed by Gabbert et al. [44] as one of the smart structure benchmark examples. A plate made of steel ( $Y=207 \mathrm{GPa}, v=0.28)$ and clamped over all four edges is considered. Material properties of the four pairs of pezopatches read: $Y=61.3 \mathrm{GPa}, v=0.3, e_{31}=e_{32}=9.6 \mathrm{C} / \mathrm{m}^{2}$. They are attached to the lower and the upper surface of the plate. The full geometry of the set-up is given in Fig. 7. The double symmetry of the structure allows modeling of only one quarter with appropriate boundary conditions. Similarly to the previous example, the patches of each pair are oppositely polarized, whereby all the patches on one side of the plate have the same polarization. The actuation of the patches is achieved by simultaneously applying the same voltage of $200 \mathrm{~V}$ to each of them.

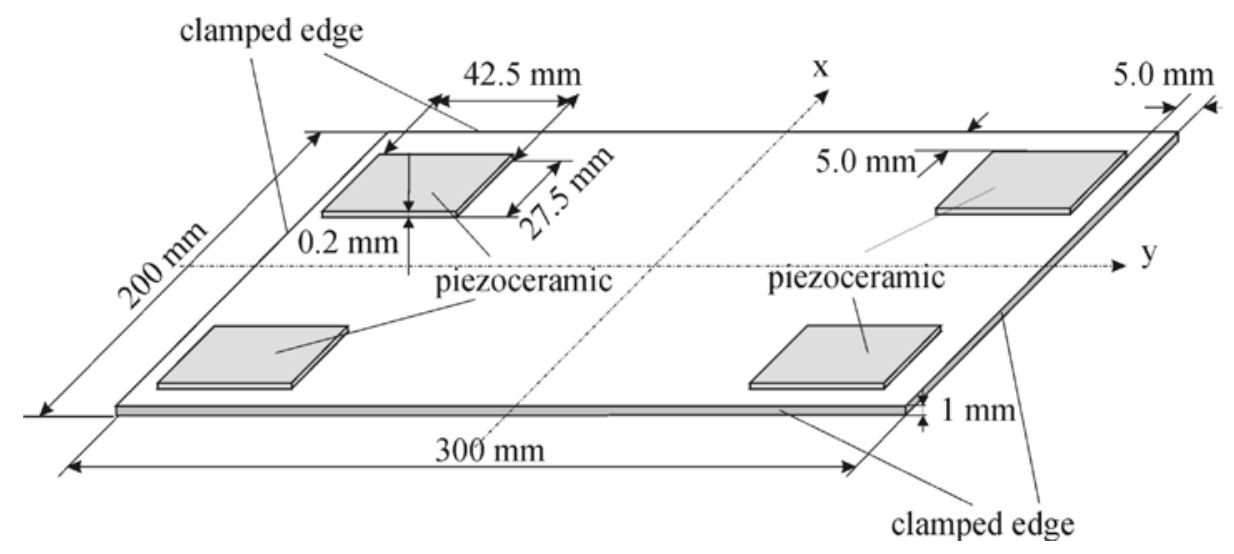

Fig. 7 Geometry of the active plate with multiple pairs of piezopatches

The FE model with the present element contains 1440 triangular elements. The diagram in Fig. 8 gives the deflection of the plate quarter along the line $x=0 \mathrm{~mm}$. The results obtained with the present element show a very high agreement with those obtained with a 9-node shell element (ACShell9) by Marinkovic [45] and the 20-node piezoelectric hexahedral element, the latter together with the experimental results reported in [44]. It can be noticed that the same displacement profiles are obtained experimentally and numerically, but that the experimentally determined displacements are smaller. This can 
be a consequence of an incomplete transfer of piezoelectrically induced loads to the carrying structure in the experiment, e.g. if there are losses in the glue layer between the patches and the passive material. As a matter of fact, Gabbert et al. [44] explain the differences by the quality of experimental set-up.

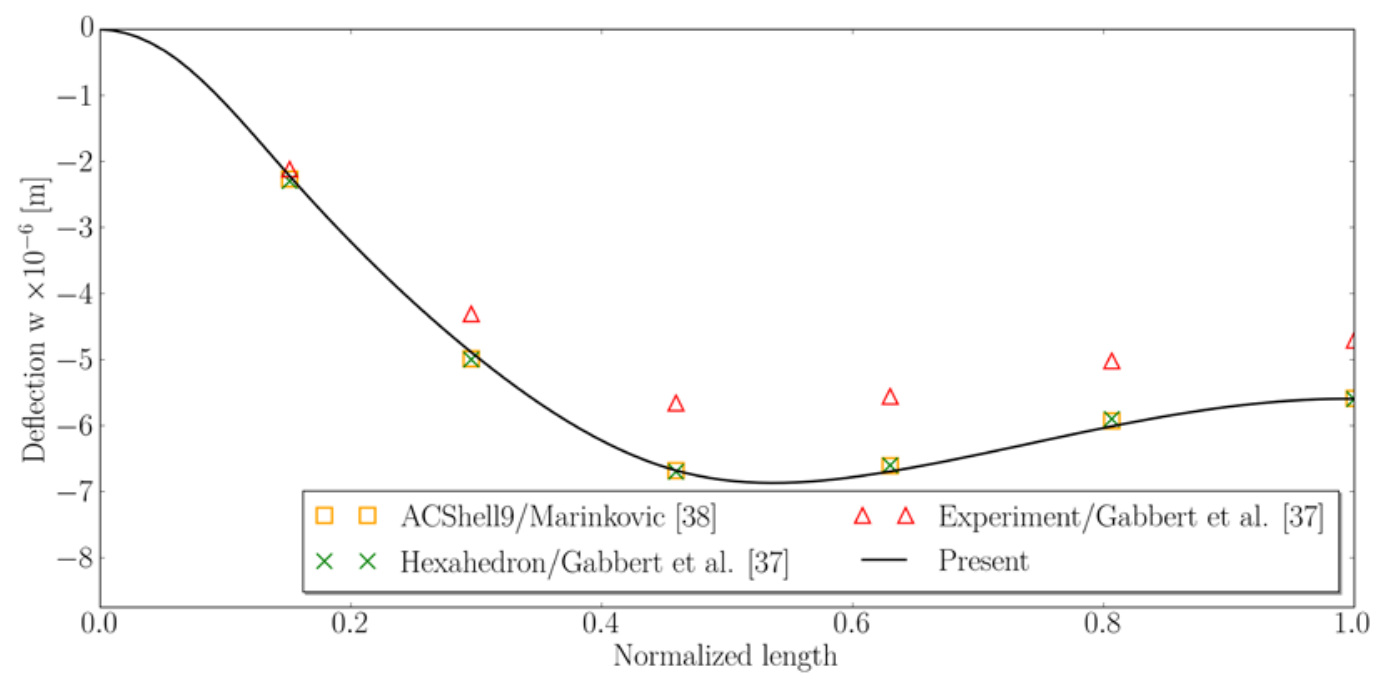

Fig. 8 Active plate deflection along $x=0$ line

\subsection{Clamped composite laminated plate with piezolayers}

The next example is chosen with the aim of demonstrating the application of the present element in modeling some features of directionally dependant material properties. For this purpose, a plate clamped over one of its edges is considered. The plate dimensions are $200 \times 195 \mathrm{~mm}$ with the overall thickness of $1.8 \mathrm{~mm}$ (Fig. 9 left). The structure host material is a laminate made of orthotropic fiber reinforced material, with the following properties in the material directions: $Y_{1}=150 \mathrm{GPa}, Y_{2}=9 \mathrm{GPa}, v_{12}=0.3$, $G_{12}=7.1 \mathrm{GPa}, G_{23}=2.5 \mathrm{GPa}$ and the properties of the piezoelectric material applied are: $Y=6.3 \mathrm{GPa}, v=0.3, e_{31}=e_{32}=22.86 \mathrm{C} / \mathrm{m}^{2}$. The thickness of each composite layer is 0.2 $\mathrm{mm}$, while the thickness of each piezoelectric layer is $0.1 \mathrm{~mm}$. The stacking sequence of the layers is $[\mathrm{p} / 30 / 90 / 30 / 90]_{s}$. Thus, it is symmetric, but 'unbalanced' in the sense that the coupling terms between bending and twisting of the plate are present in the ABD 
matrix determined with respect to the structure reference direction, which is the global x-direction. In other words, a load that would cause bending but no twisting deformation of the considered structure made of an isotropic material, now generates twisting as well.

Two load cases are considered - a purely mechanical excitation and an actuation of the piezolayers by a constant electric voltage. Linear and geometrically nonlinear computations are performed and in order to capture the local rigid-body rotations adequately in the nonlinear analysis, the structure is discretized by 200 elements. For the comparison purposes, the same examples are computed in Abaqus using the S3 element and the same mesh.

In the first case, the plate is exposed to three concentrated forces, two of which have a magnitude of $12 \mathrm{~N}$ and act at the corner points of the edge opposite to the clamped edge (points $\mathrm{A}$ and $\mathrm{C}$ in Fig. 9), while the third force of $24 \mathrm{~N}$ acts at the mid-point of the same edge (point B in Fig. 9).
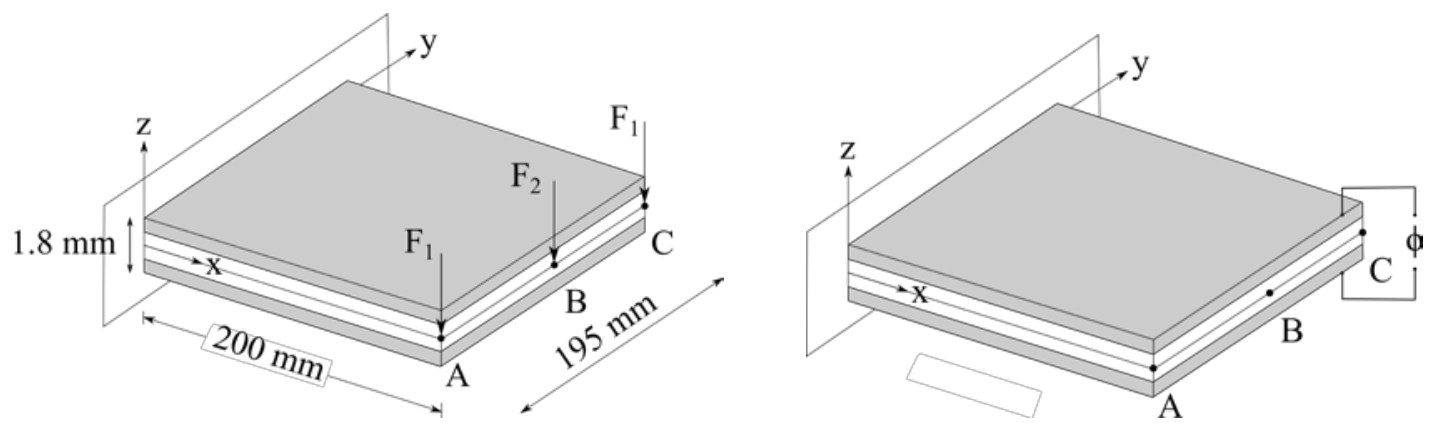

Fig. 9 Geometry and boundary conditions of the piezolaminated semicircular shell

Since both bending and twisting of the shell are induced, the vertical displacement (in the global z-direction), $w$, is observed at the same points at which the forces act. The results by the present element and from Abaqus for both linear and geometrically nonlinear analyses are summarized in Table 1 . A very good agreement between the results can be noticed. 
Table 1 Mechanical excitation of the clamped composite plate - free edge displacements

\begin{tabular}{|c|c|c|c|c|}
\hline \multirow{2}{*}{\multicolumn{2}{|c|}{ Mechanical excitation }} & Point A & Point B & Point C \\
\hline & & \multicolumn{3}{|c|}{ Linear analysis } \\
\hline \multirow{5}{*}{$\begin{array}{c}\text { Displacement } \\
\text { w [mm] }\end{array}$} & Abaqus S3 & -50.93 & -36.75 & -22.9 \\
\hline & Present & -50.94 & -36.8 & -22.9 \\
\hline & & \multicolumn{3}{|c|}{ Geometrically nonlinear analysis } \\
\hline & Abaqus S3 & -44.81 & -33.19 & -23.55 \\
\hline & Present & -44.8 & -33.22 & -23.58 \\
\hline
\end{tabular}

In the second case the oppositely polarized piezoelectric layers of the same structure are subjected to a constant voltage of $1000 \mathrm{~V}$. The relatively large value of electric voltage is chosen in order to produce sufficiently large deformation that would justify the geometrically nonlinear analysis. The induced bending moments uniformly distributed over all shell edges cause again bending and twisting of the structure. In order to compute this case in Abaqus the equivalent bending moment uniformly distributed over the plate free edges (Fig. 10) is first computed and directly applied in Abaqus.

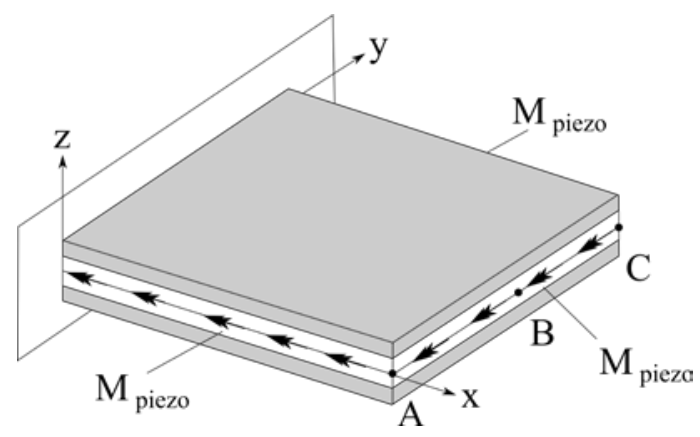

Fig. 10 Uniformly distributed edge bending moments due to piezoelectric excitation

It should be emphasized that the orientation of the piezoelectric loads depends on the current structure configuration. This effect is not recognized in the linear analysis, but it is present in the geometrically nonlinear analysis. Therefore, for the geometrically nonlinear analysis in Abaqus the moments are set to be of the 'follower type', whereby the nodal moments follow the nodal rotations. On the other hand, the effect is automatically included in the presented formulation where the induced moments depend 
on the element rotation. A very good agreement of the linear results by the two elements can be seen in Table 2, while slight differences between the geometrically nonlinear results can be noticed (less than $2 \%$ ), which is to be attributed to the above explained difference in how the 'follower force' property of the induced loads is accounted for in Abaqus and in the present formulation.

Table 2 Voltage excitation of the clamped composite plate - free edge displacements

\begin{tabular}{|c|c|c|c|c|}
\hline \multicolumn{2}{|c|}{ Voltage excitation } & Point A & Point B & Point C \\
\cline { 4 - 5 } & Abaqus S3 & 91.78 & -48.0 & 20.64 \\
\cline { 2 - 5 } & Present & 91.84 & 48.0 & 20.64 \\
\cline { 2 - 5 } $\begin{array}{c}\text { Displacement } \\
w[\mathrm{~mm}]\end{array}$ & & \multicolumn{3}{|c|}{ Geometrically nonlinear analysis } \\
\cline { 2 - 5 } & Abaqus S3 & 83.15 & 45.89 & 21.72 \\
\cline { 2 - 5 } & Present & 83.59 & 46.3 & 22.1 \\
\hline
\end{tabular}

\subsection{Piezo-laminated semicircular arch}

In the next example a curved structured is considered. It is a semicircular shell clamped at one end and free at the other, Fig. 11 . The radius of the mid-surface is $R=318.31 \mathrm{~mm}$, the width $w=50.8 \mathrm{~mm}$ and the overall thickness $h=6.35 \mathrm{~mm}$. The structure consists of three layers. The passive mid-layer is metallic ( $Y=68.95 \mathrm{GPa}, v=0.3, \rho=7750 \mathrm{~kg} / \mathrm{m}^{3}$ ) and has the thickness of $5.842 \mathrm{~mm}$, while the outer two are piezoelectric layers $(Y=63$ GPa, $\left.v=0.3, \rho=7600 \mathrm{~kg} / \mathrm{m}^{3}, e_{31}=e_{32}=16.11 \mathrm{C} / \mathrm{m}^{2}, d_{33}=1.65 \cdot 10^{-8} \mathrm{~F} / \mathrm{m}\right)$, each with the thickness of $0.254 \mathrm{~mm}$.

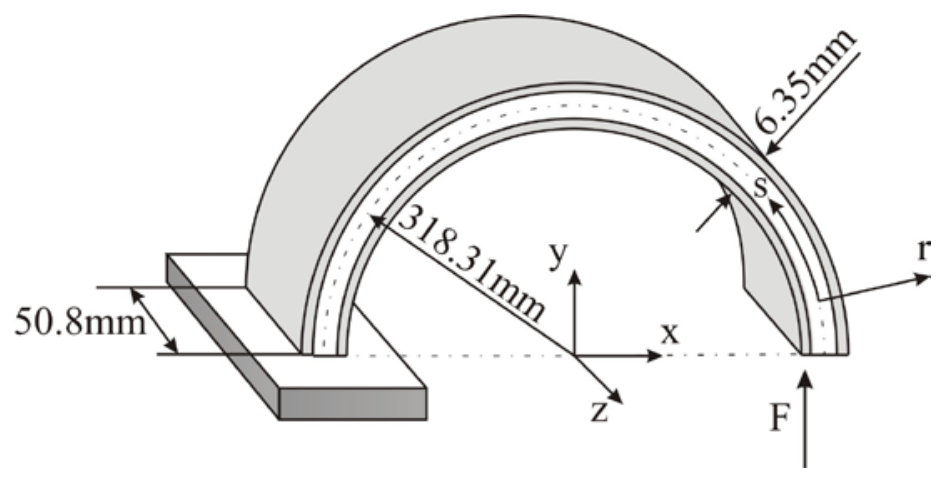

Fig. 11 Geometry and boundary conditions of the piezolaminated semicircular arch 
This example was originally proposed by Tzou and Ye [46] and was further modified by Zhang [47] so that static and dynamic, linear and geometrically nonlinear analyses are covered. As an excitation, a single force F acting at the center of the free arch tip (Fig. 11) is applied and the piezoelectric layers are used as sensors. In the present study, the structure was meshed by 160 triangular elements.

First, the force magnitude is set to $100 \mathrm{~N}$ and a static response is considered in a geometrically nonlinear analysis. As a representative structural response, the displacements of the free arch tip in the hoop (s) and radial (r) directions are observed together with the voltage of the inner piezoelectric layer. The results for the displacements obtained with the presented element and formulation are compared with those from Zhang [47] and Abaqus S3 element. The element used by Zhang [47] (denoted as SH851URI) is an 8-node shell element with five nodal mechanical degrees of freedom and one electrical degree of freedom per piezolayer and it applies uniformly reduced integration. The results for the displacements by all three elements are depicted in Fig. 12 and show very good agreement.

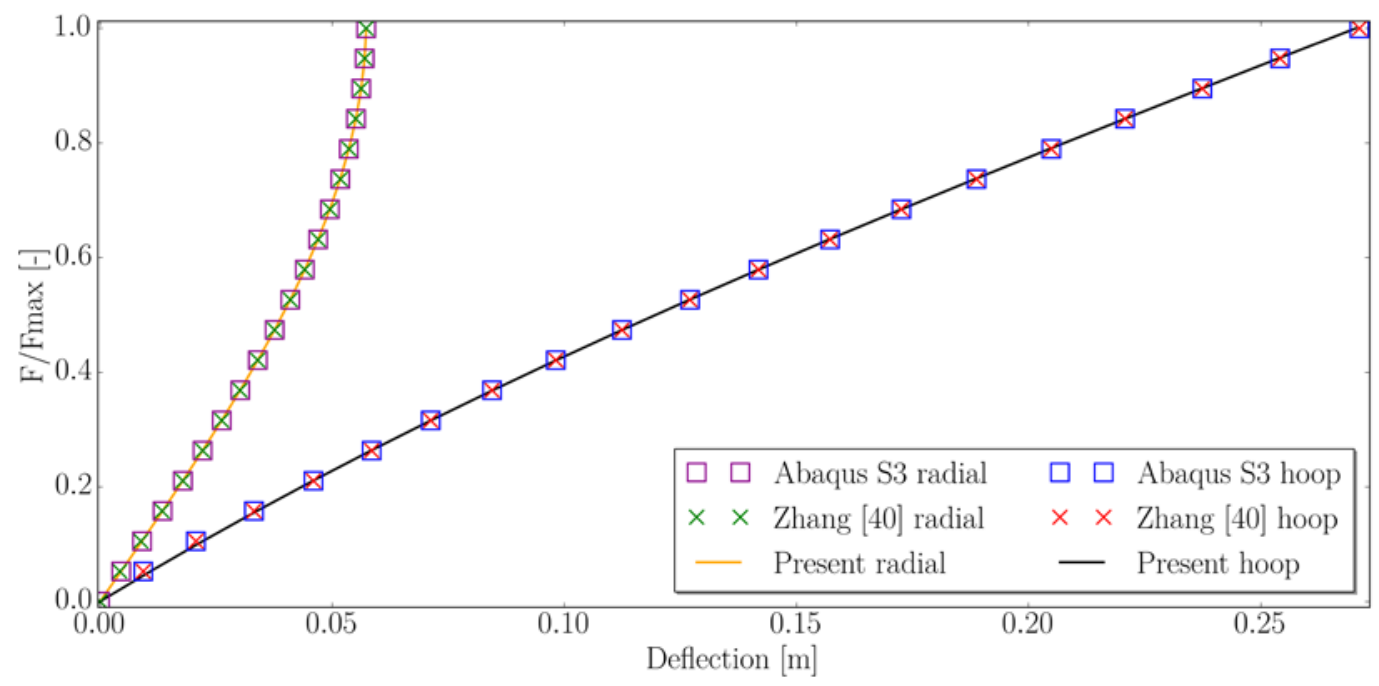

Fig. 12 Geometrically nonlinear static case - displacements of the free arch tip in the hoop and radial directions 
On the other hand, in Fig. 13 one may notice a minor difference between the results for the induced electric potential in the inner piezolayer obtained by the present formulation and reported by Zhang [47]. Beside the different element type and discretization (Zhang used 1 element across the width and 10 elements in the hoop direction), this difference can also be attributed to the fact that Zhang used the rigorous geometrically nonlinear formulation, whereas the co-rotational formulation used in this work implies that the element behavior with respect to the local reference frame remains linear. In addition, using the linear triangular element to model a curved structure results in a faceted representation of the geometry and this may affect the distribution of bending moments. This influence can also gain in importance over the course of deformation in a geometrically nonlinear analysis.

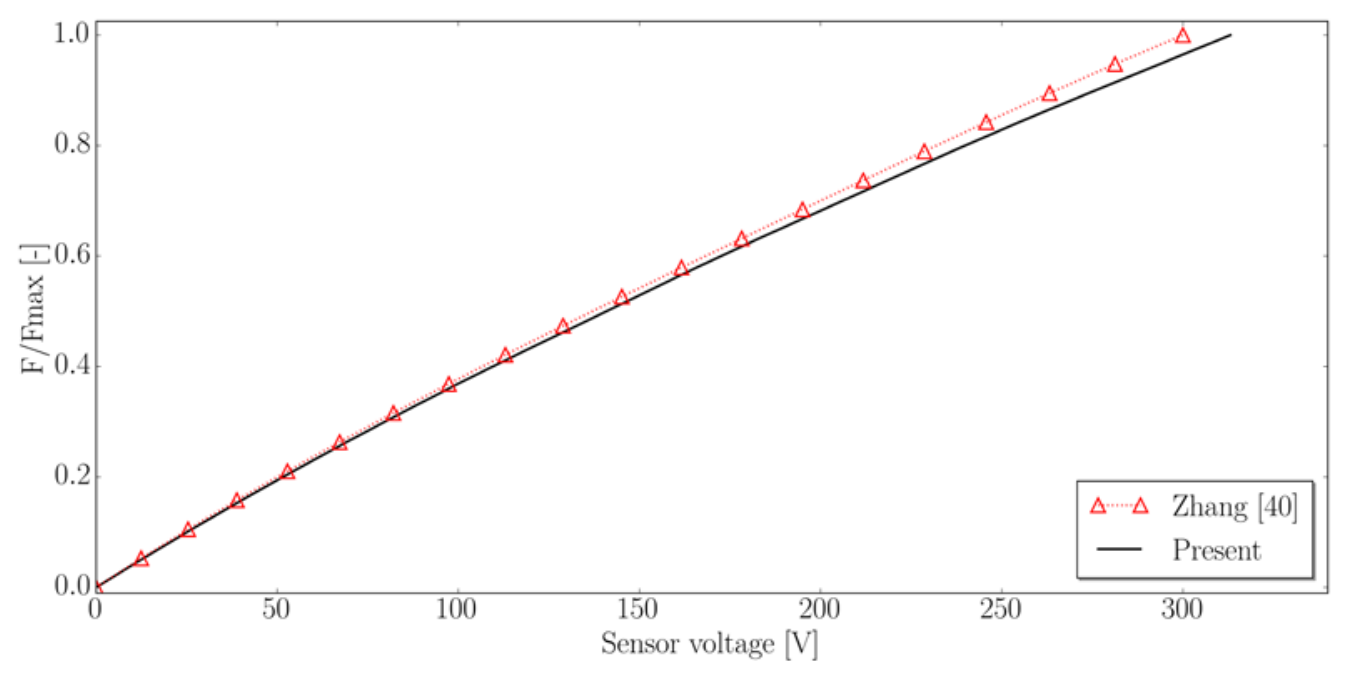

Fig. 13 Geometrically nonlinear static case - sensor voltage of the inner piezolayer

For dynamic analysis, the force is applied as a step function with the magnitude of $50 \mathrm{~N}$ and the response of the structure is observed in a time interval of $1 \mathrm{~s}$. The same quantities as in the static case are observed, i.e. the displacements in the radial and hoop directions together with the sensor voltage of the inner piezolayer. Linear and geometrically nonlinear computations are performed, whereby the time-step of $10^{-3} \mathrm{~s}$ is used for the linear analysis and $10^{-4} \mathrm{~s}$ for the nonlinear analysis. The results obtained by 
the present element and formulation and those reported by Zhang [47] are summarized in diagrams in Figs. 14-16.

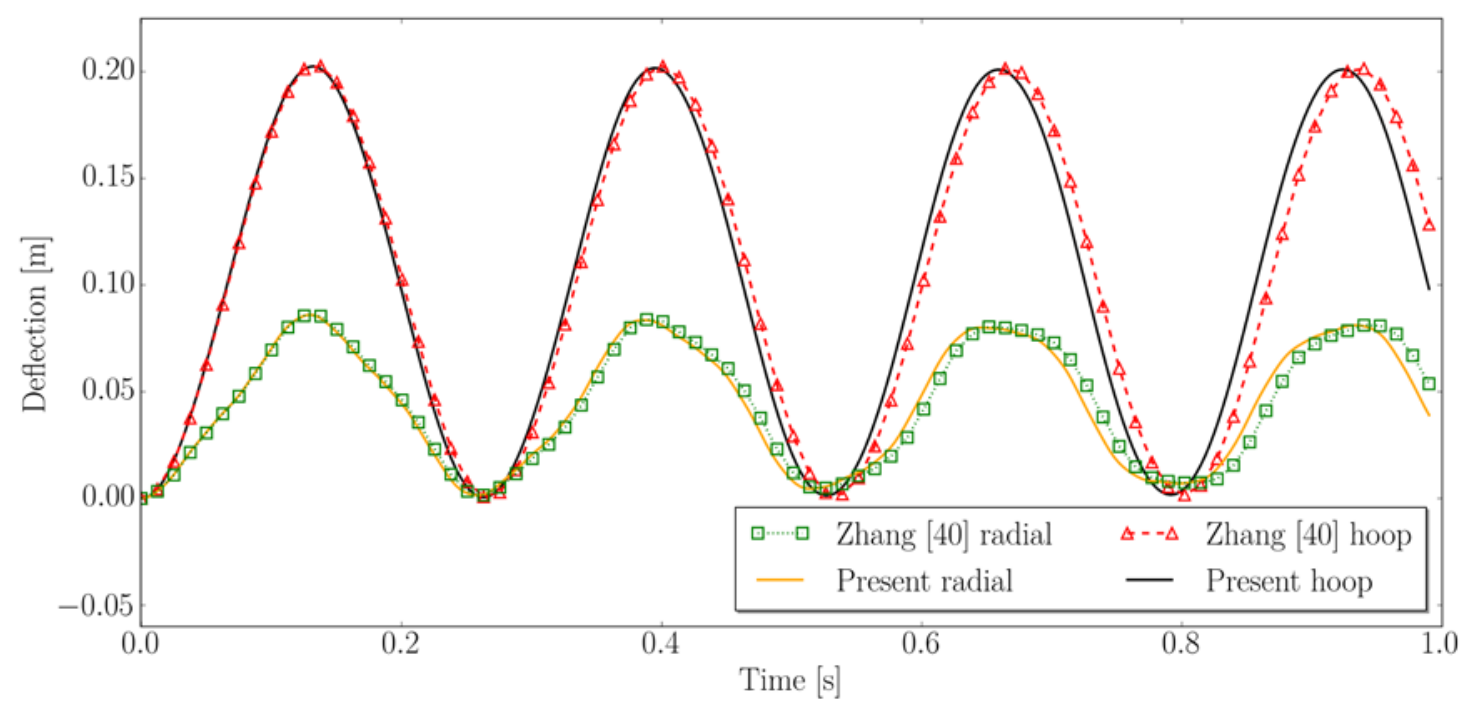

Fig. 14 Linear analysis - displacement of the arch tip in radial and hoop directions

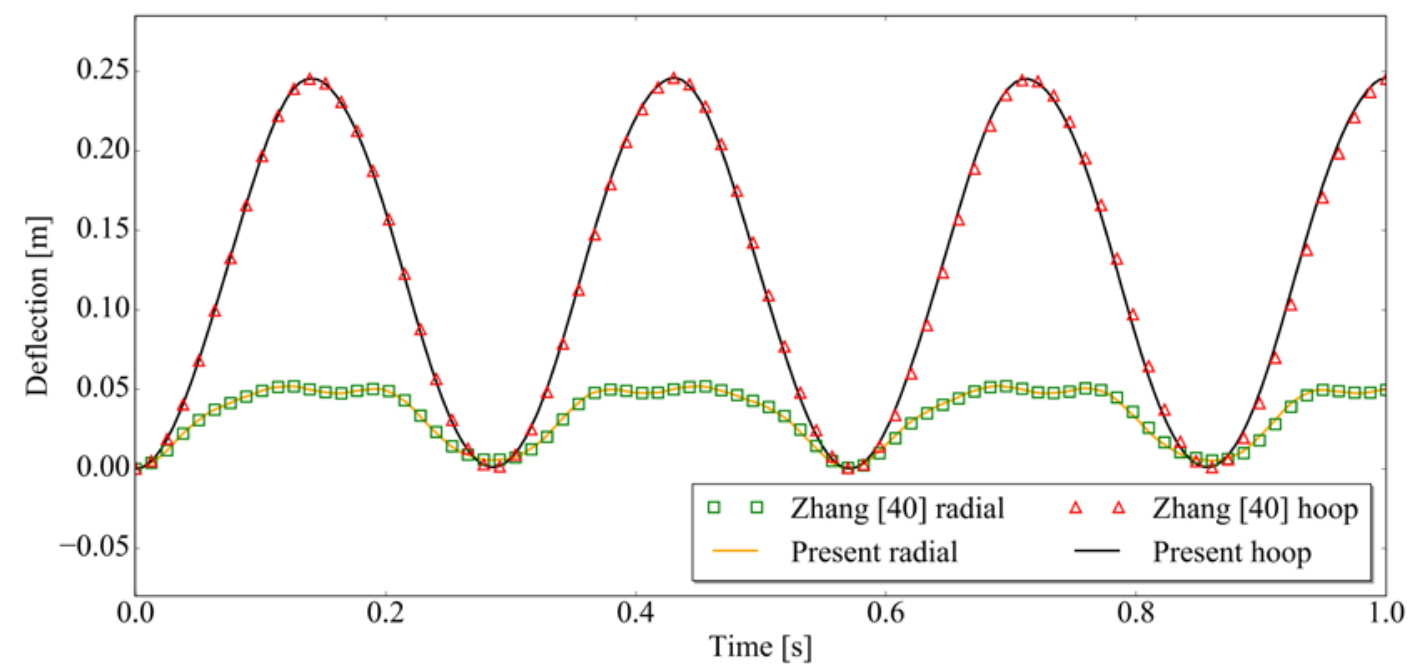

Fig. 15 Nonlinear analysis - displacement of the arch tip in radial and hoop directions 


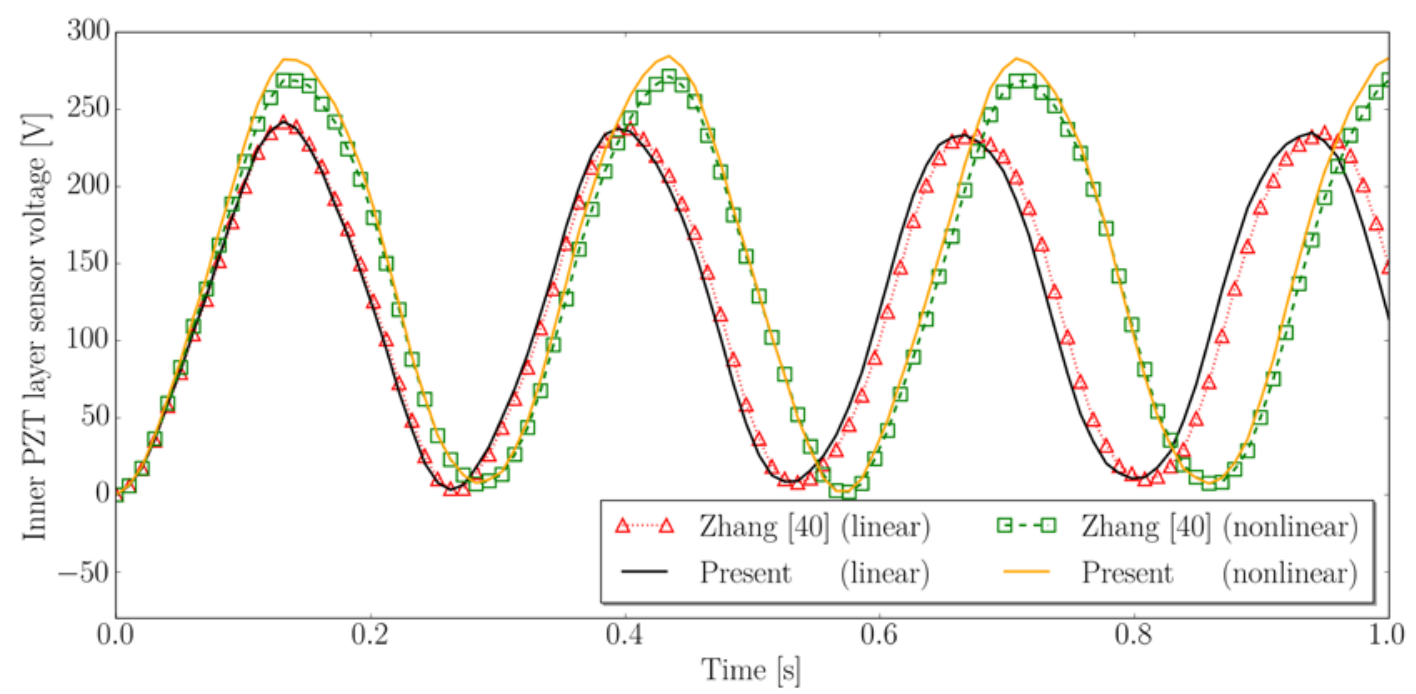

Fig. 16 Sensor voltage of the inner piezolayer - linear and nonlinear result

Observing the linear results for displacements (Fig. 14), one may notice that the structural response is dominated by the first eigenmode (with the present element frequency $f_{1}=3.755 \mathrm{~Hz}$ ), whereby the radial deflection also reveals presence of a higher eigenmode in the structural response and that is the third eigenmode (frequency $\mathrm{f}_{3}=11.9$ $\mathrm{Hz}$ ). The second eigenmode is normal to the applied force and, hence, not a part of the structural response. In the first eigenmode, the displacement of the structure's free end has significant components in both the radial (r) and hoop (s) directions (Fig. 17, left). Consequently, this frequency is dominant in the both observed displacements. In the third mode, the free tip displacement has a relatively small component in the hoop direction and a significant component in the radial direction (Fig. 17, right). This explains why in the linear transient displacement in the hoop direction only one frequency is perceived visually from the diagram (the third is also present, but not so obvious), whereas in the radial displacement a mixture of the two eigenfrequencies can be visually recognized. 


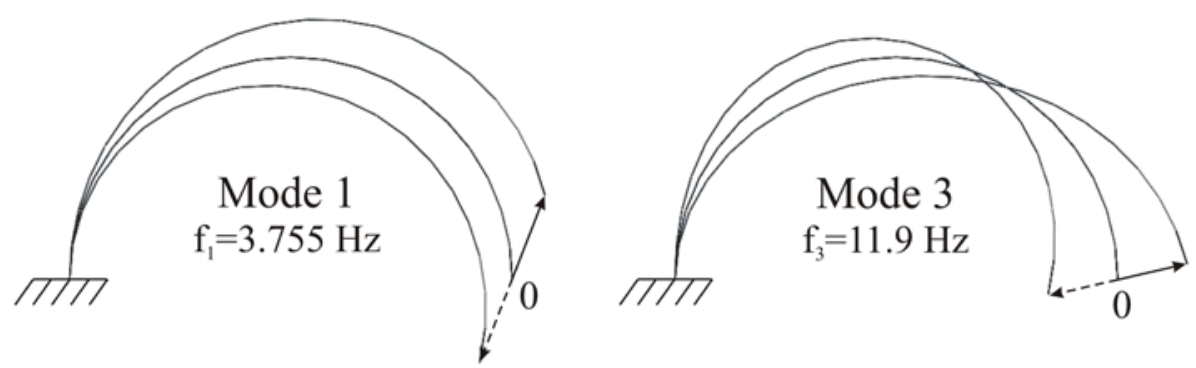

Fig. 17 The first and third mode shapes of the semicircular arch

4.5 Simply supported plate - shape control and actuated dynamics

In the final example, a plate simply supported over two parallel edges is considered, Fig. 18. Its dimensions are $200 \times 200 \times 1 \mathrm{~mm}$. The host material is a composite laminate with the following properties in the material directions: $Y_{1}=132.28 \mathrm{GPa}, Y_{2}=10.76 \mathrm{GPa}$, $v_{12}=0.24, G_{12}=5.65 \mathrm{GPa}, G_{23}=3.61 \mathrm{GPa}$. The piezoelectric material properties are: $Y=63$ GPa, $v=0.3, \rho=7600 \mathrm{~kg} / \mathrm{m}^{3}, e_{31}=e_{32}=22.86 \mathrm{C} / \mathrm{m}^{2}$. The thickness of each layer is 0.25 $\mathrm{mm}$. The sequence of layers with respect to the structure reference direction (x-axis) is [p/45/-45/0]s. Static and dynamic analyses are performed using a mesh with 200 elements.

In the static analysis, a similar scenario for the shape control is used as by Kioua and Mirza [48]. The shape control example proposed by Kioua and Mirza was resolved by means of the Ritz method. It has been used by a number of authors as a benchmark example and all the authors reported a difference between the Ritz solution obtained by Kioua and Mirza and the FEM results. A detailed analysis of this difference was given by Marinkovic et al. [49]. For this reason, the authors of this paper decided to offer a slightly modified version of the example to the research community. Hence, the plate is first exposed to a single force, $\mathrm{F}=30 \mathrm{~N}$, perpendicular to the surface of the plate and acting at its centroid, Fig. 18. Upon the deformation, the oppositely polarized piezoelectric layers are supplied with the slowly increasing electric voltage and the 
deformational behavior is observed. As already explained, the electric voltage gives rise to bending moments uniformly distributed over the plate edges.

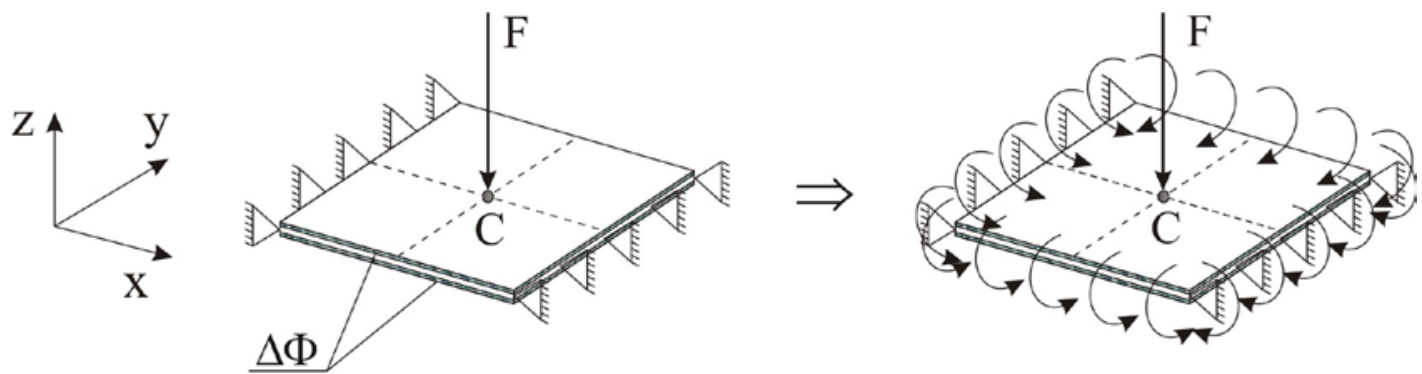

Fig. 18 Boundary conditions of the piezo-laminated simply supported plate

The bending moments induced through the inverse piezoelectric effect tend to reduce the plate deflection. Clearly, it is impossible to recover exactly the original configuration (zero deflection over the whole surface) in this manner, but a configuration very close to the original one is obtained for the voltage of $137.5 \mathrm{~V}$. This can be see in Fig. 19 which depicts deflection along the the x-direction through the plate centroid $\mathrm{C}$ for different values of the electric voltage supplied to the piezopatches. The results are obtained in a linear analysis as deformations are rather small and for the purpose of comparison the results obtained in Abaqus with the S3 element and the precomputed edge distributed bending moments are used. Obviously, the two elements yield practically congruent results. 


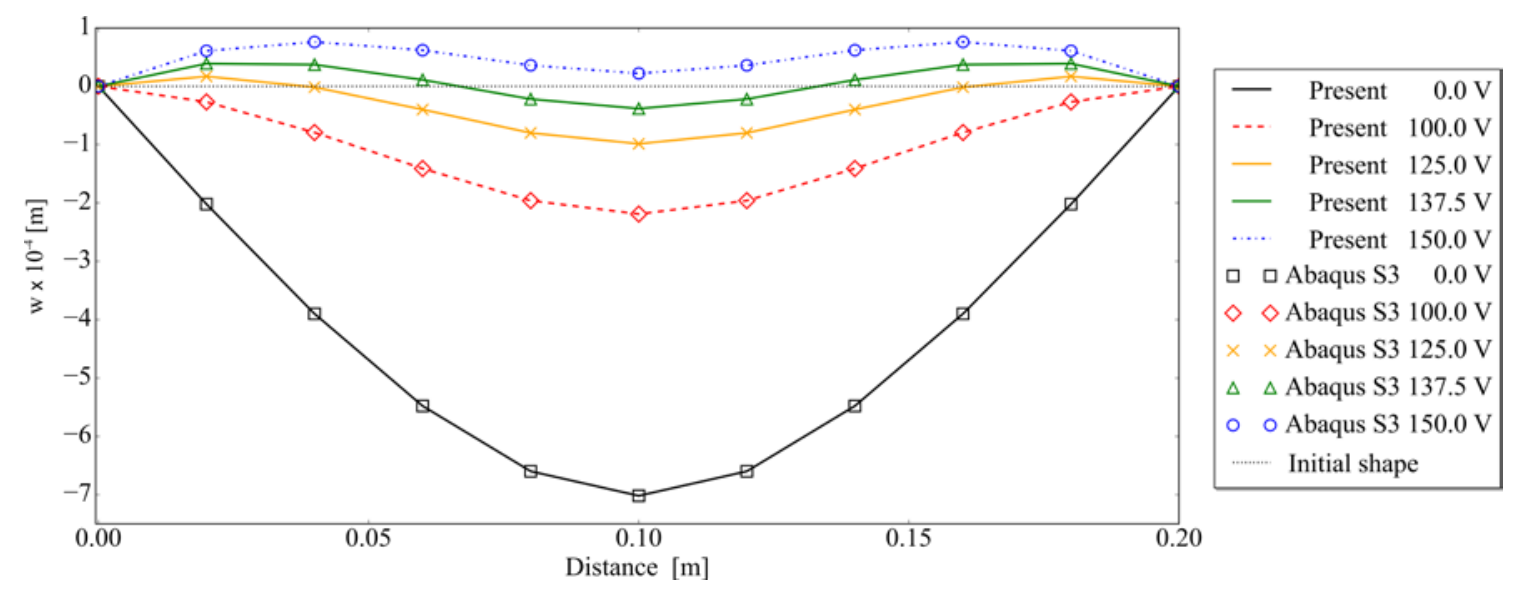

Fig. 19 Simply supported plate - deflection along the x-direction through the plate centroid

Finally, deformation of the same structure exposed to a dynamic excitation is considered. The time dependent excitation is electric voltage in a sine form with the amplitude of $250 \mathrm{~V}$ and frequency of $50 \mathrm{~Hz}$. Linear and geometrically nonlinear analyses are performed. The follower nature of the induced bending moments is considered in Abaqus in exactly the same manner as explained in the $3^{\text {rd }}$ example with the plate clamped over one edge. The deflection of the point $\mathrm{C}$ is observed in a time interval of $0.04 \mathrm{~s}$ and a very good agreement between the results obtained with the present element and in Abaqus can be seen in Fig. 20.

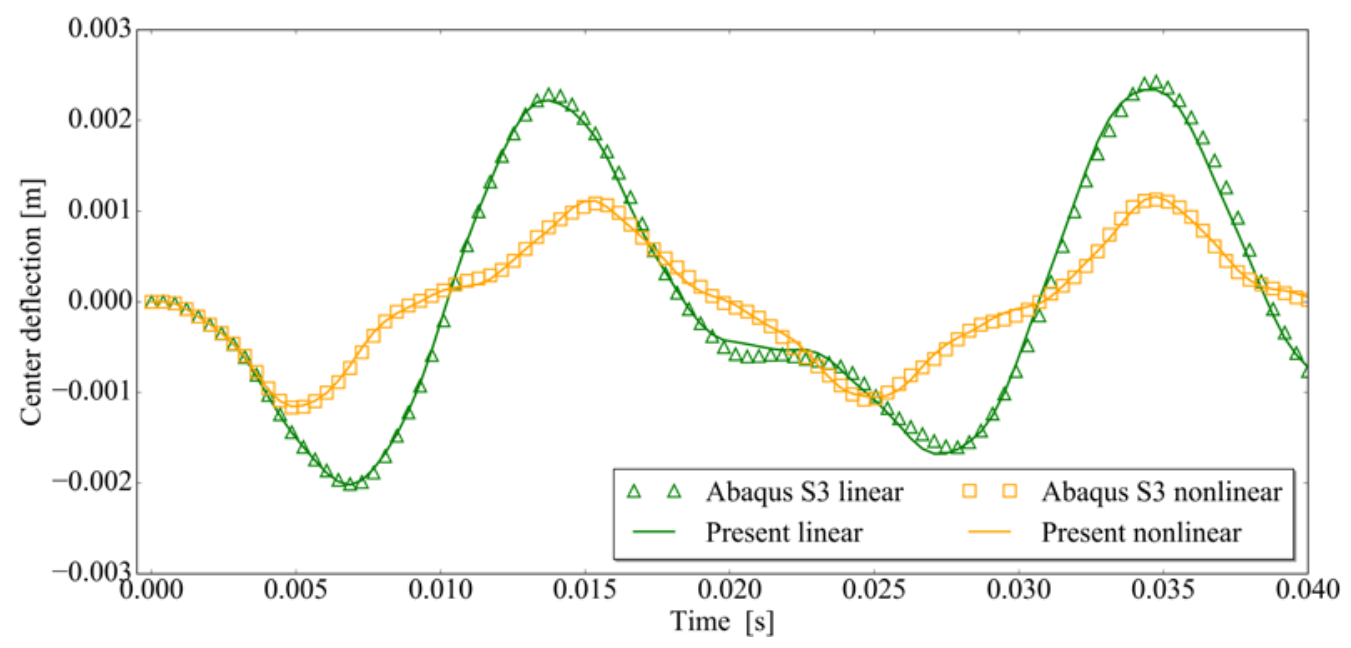

Fig. 20 Plate centroid deflection as a response to time dependent electric voltage 


\section{CONCLUSIONS}

Combining the exquisite mechanical properties of composite laminates with the multifunctional character of piezoelectric materials enables new lightweight design solutions with integrated sensing and actuating. Such a material system offers vast options to improve the structural safety, reliability and robustness through adaptive behavior. Numerical tools that enable efficient and accurate simulations of such material systems are of great interest to the engineering community.

The choice to develop a 3-node shell element was driven by the element's high meshing ability and numerical efficiency. However, linear elements are generally known for relatively stiff behavior. To address this issue, the enhancements in the form of strain-smoothing technique and discrete shear gap were applied in the element formulation. Furthermore, the co-rotational formulation was used to extend the applicability of the element into the realm of geometrically nonlinear analysis. The formulation accounts for rigid-body motion element-wise. Hence, finer meshes are favored in order to capture the local rigid-body rotations adequately. But this is fully in compliance with the fact that low-order elements demand finer meshes anyway.

The considered examples cover static and dynamic cases, with the piezolayers used as both actuators and sensors, and linear as well as geometrically nonlinear analyses were performed. As reference solutions, the experimental and numerical results available in the literature were used, but also numerical results from commercially available FEM program Abaqus. Though examples are more of academic nature, they not only prove the applicability of developed element and formulation, but also expose a few important aspects of modeling thin-walled piezoelectric structures. It was shown that geometrically nonlinear effects may easily become important in the behavior of the considered structures, which is certainly related to the load level, but also to the 
kinematic boundary conditions. This is particularly valid in the cases where, over the course of deformation, a thin-walled structure rapidly changes the way it resists the external loads, for instance from plate dominated behavior to shell (membrane) dominated behavior. Also, one of the aspects is the follower nature of the loads induced by the inverse piezoelectric effect and the necessity to account for it.

In the future work, in-plane polarized piezoelectric materials should be given attention, particularly composite materials with piezoelectric fibers. Also, the element should be extended to cover the materially nonlinear effects in the piezoelectric coupling. The co-rotational formulation offers a solid base for this as it separates geometric from material nonlinearities.

\section{References}

1. Gabbert U, Tzou HS., editors. Smart Structures and Structronic Systems. Amsterdam: Kluwer Academic Publishers, 2000.

2. Marinkovic D, Nestorovic T, Marinkovic Z, Trajkov M, Modelling and simulation of piezoelectric adaptive structures. Transactions of FAMENA 2012;36(1):25-34.

3. Aridogan U, Basdogan I. A review of active vibration and noise suppression of plate-like structures with piezoelectric transducers. Journal of Intelligent Material Systems and Structures 2015; 26(12):1455-1476

4. Li FM, Yao G, Zhang Y. Active control of nonlinear forced vibration in a flexible beam using piezoelectric material. Mechanics of Advanced Materials and Structures 2016;23(3):311-317. 
5. Masmoudi S, Mahi AE, Turki S, Use of piezoelectric as acoustic emission sensor for in situ monitoring of composite structures. Composites Part B: Engineering 2015;80:307-320.

6. Sha F, Cheng X, Li S, Xu D, Huang S, Liu R, Li Z, Xie X, Guo X. Nondestructive evaluation on strain sensing capability of piezoelectric sensors for structural health monitoring. Research in Nondestructive Evaluation 2017; 28(2):61-75.

7. Zhang LW, Song ZG, Liew KM, Optimal shape control of CNT reinforced functionally graded composite plates using piezoelectric patches. Composites Part B: Engineering 2016;85:140-149.

8. Zhang SQ, Li YX, Schmidt R. Active shape and vibration control for piezoelectric bonded composite structures using various geometric nonlinearities. Composite Structures 2015;122:239-249

9. Wang BT, Rogers CA. Laminate plate theory for spatially distributed induced strain actuators. Journal of Composite Materials 1991;25(4):433-452.

10. Bohlooly M, Mirzavand B. Closed form solutions for buckling and postbuckling analysis of imperfect laminated composite plates with piezoelectric actuators. Composites Part B: Engineering 2015;72:21-29

11. Ansari R, Pourashraf T, Gholami R, Shahabodini A. Analytical solution for nonlinear postbuckling of functionally graded carbon nanotube-reinforced composite shells with piezoelectric layers. Composites Part B: Engineering 2016;90:267-277.

12. Benjeddou A. Advances in piezoelectric finite element modeling of adaptive structural elements: a survey. Computers and Structures 2000;76:347-363. 
13. Klinkel S, Wagner W. A piezoelectric solid shell element based on a mixed variational formulation for geometrically linear and nonlinear applications. Computers and Structures 2008;86(1-2):38-46.

14. Lee S, Cho BC, Park HC, Goo NS, Yoon KJ. Piezoelectric actuator-sensor analysis using a three-dimensional assumed strain solid element, Journal of Intelligent Material Systems and Structures 2004;15(5):329-338.

15. Willberg C, Gabbert U. Development of a three-dimensional piezoelectric isogeometric finite element for smart structure applications. Acta Mechanica 2012;223:1837-1850.

16. Li E, He ZC, Chen L, Li B, Xu X, Liu GR. An ultra-accurate hybrid smoothed finite element method for piezoelectric problem. Engineering Analysis with Boundary Elements 2015;50:188-197.

17. Gabbert U, Köppe H, Seeger F, Berger H. Modeling of smart composite shell structures. Journal of Theoretical and Applied Mechanics 2002;3(40):575-593.

18. Simoes Moita JM, Mota Soares CM, Mota Soares CA. Geometrically non-linear analysis of composite structures with integrated piezoelectric sensors and actuators. Composite structures 2002;57:253-61

19. Nguyen-Xuan H., Liu GR, Nguyen-Thoi T, Nguyen-Tran C. An edge-based smoothed finite element method for analysis of two-dimensional piezoelectric structures. Smart Materials and Structures 2009;18(6):065015 (12pp)

20. Zemčík R, Rolfes R, Rose M, Tessmer J. High performance four node shell element with piezoelectric coupling for the analysis of smart laminated structures. International journal for numerical methods in engineering 2007;70(8):934-961. 
21. Lentzen S, Klosowski P, Schmidt R. Geometrically nonlinear finite element simulation of smart piezolaminated plates and shells. Smart Materials and Structures 2007;16:2265-2274

22. Marinkovic D, Köppe H, Gabbert U. Degenerated shell element for geometrically nonlinear analysis of thin-walled piezoelectric active structures. Smart Materials and Structures 2008;17(1):1-10.

23. Kögl M, Bucalem ML. Analysis of smart laminates using piezoelectric MITC plate and shell elements. Computers and Structures 2005;83(15-16):1153-1163

24. Marinkovic D, Marinkovic Z. On FEM modeling of piezoelectric actuators and sensors for thin-walled structures. Smart Structures and Systems 2012;9(5):411-426.

25. Nestorovic T, Shabadi S, Marinkovic D, Trajkov M. User defined finite element for modeling and analysis of active piezoelectric shell structures. Meccanica 2014;49(8):1763-1774.

26. Phung-Van P, De Lorenzis L, Thai $\mathrm{CH}$, Abdel-Wahab M, Nguyen-Xuan $\mathrm{H}$. Analysis of laminated composite plates integrated with piezoelectric sensors and actuators using higher-order shear deformation theory and isogeometric finite elements. Computational Materials Science 2015;96(B):495-505.

27. Carrera E, Theories and finite elements for multilayered plates and shells: A unified compact formulation with numerical assessment and benchmarking, Archives of Computational Methods in Engineering 2003;10:215-296.

28. Cinefra M, Carrera E, Valvano S. Variable kinematic shell elements for the analysis of electro-mechanical problems. Mechanics of Advanced Materials and Structures 2015;22(1-2):77-106. 
29. Cinefra M, Valvano S, Carrera E. A layer-wise MITC9 finite element for the freevibration analysis of plates with piezo-patches. International Journal of Smart and Nano Materials 2015;6(2):84-104.

30. Milazzo A. Unified formulation for a family of advanced finite elements for smart multilayered plates. Mechanics of Advanced Materials and Structures 2016;23(9):971-980.

31. Bletzinger KU, Bischoff M, Ramm E. A unified approach for shear-locking-free triangular and rectangular shell finite elements. Computers and Structures 2000;75(3):321-334.

32. Nguyen-Thoi T, Phung-Van P, Thai-Hoang C, Nguyen-Xuan H. A Cell-based Smoothed Discrete Shear Gap method (CS-DSG3) using triangular elements for static and free vibration analyses of shell structures. International Journal of Mechanical Sciences 2013;74:32-45.

33. Chrisfield MA, Moita GF. A co-rotational formulation for 2-D continua including incompatible modes. International Journal for Numerical Methods in Engineering 1996;39(15):2619-2633.

34. Felippa C, Haugen B. A unified formulation of small-strain corotational finite elements: I. theory. Computer Methods in Applied Mechanics and Engineering 2005;194:2285-2335.

35. Nguyen VA, Zehn M, Marinković D. An efficient co-rotational FEM formulation using a projector matrix. Facta Universitatis Series Mechanical Engineering 2016;14(2):227-240.

36. Berthelot JM. Composite Materials: Mechanical Behavior and Structural Analysis. New York: Springer-Verlag, 1999. 
37. Ikeda T. Fundamentals of Piezoelectricity. New York: Oxford University Press Inc, 1996

38. Marinković D, Köppe H, Gabbert U. Accurate modeling of the electric field within piezoelectric layers for active composite structures. Journal of Intelligent Material Systems and Structures 2007;18(5):503-513.

39. Marinković D, Köppe H, Gabbert U. Aspects of modeling piezoelectric active thinwalled structures. Journal of Intelligent Material Systems and Structures 2009;20(15):1835-1844.

40. Allik H, Hughes TJR. Finite element method for piezoelectric vibration. International Journal for Numerical Methods in Engineering 1970;2(2):151-157

41. Bathe KJ. Finite Element Procedures, New York: Prentice Hall. 1996.

42. Argyris J. An excursion into large rotations. Computer Methods in Applied Mechanics and Engineering 1982;32(1-3):85-155.

43. Gupta VK, Seshu P, Kurien Isaac K. Finite element and experimental investigation of piezoelectruc actuated smart shells. AIAA Journal 2004;42(10):2112-2123.

44. Gabbert U, Görnandt A, Köppe H. Benchmark problems for the analysis of piezothermoelastic smart structures. Proceedings of NAFEMS Seminar: Finite element applications for adaptive structural elements. Magdeburg, Germany, November 1998, paper 5, 12 pages.

45. Marinković D. A New Finite Composite Shell Element for Piezoelectric Active Structures. Ph.D. thesis. Otto-von-Guericke Universität Magdeburg, FortschrittBerichte VDI, Reihe 20: Rechnerunterstützte Verfahren, Nr. 406, Düsseldorf, 2007.

46. Tzou HS, Ye R. Analysis of piezoelastic structures with laminated piezoelectric triangle shell elements. AIAA Journal 1996;34:110-115. 
47. Zhang S. Nonlinear FE Simulation and Active Vibration Control of Piezoelectric Laminated Thin-Walled Smart Structures. Ph.D. thesis. RWTH Aachen University, Aachen, 2014.

48. Kioua H, Mirza S. Piezoelectric induced bending and twisting of laminated composite shallow shells. Smart Materials and Structures 2000;9(4):476-484.

49. Marinkovic D, Marinkovic Z. FEM and Ritz method - a piezoelectric active shell case study. Transactions of FAMENA 2011;35(3):39-48. 